\title{
Tetrapyrrole Signaling in Plants
}

\author{
Robert M. Larkin*
}

Key Laboratory of Horticultural Plant Biology (Ministry of Education), College of Horticulture and Forestry Sciences, Huazhong Agricultural University, Wuhan, China

Tetrapyrroles make critical contributions to a number of important processes in diverse organisms. In plants, tetrapyrroles are essential for light signaling, the detoxification of reactive oxygen species, the assimilation of nitrate and sulfate, respiration, photosynthesis, and programed cell death. The misregulation of tetrapyrrole metabolism can produce toxic reactive oxygen species. Thus, it is not surprising that tetrapyrrole metabolism is strictly regulated and that tetrapyrrole metabolism affects signaling mechanisms that regulate gene expression. In plants and algae, tetrapyrroles are synthesized in plastids and were some of the first plastid signals demonstrated to regulate nuclear gene expression. In plants, the mechanism of tetrapyrroledependent plastid-to-nucleus signaling remains poorly understood. Additionally, some of experiments that tested ideas for possible signaling mechanisms appeared to produce conflicting data. In some instances, these conflicts are potentially explained by different experimental conditions. Although the biological function of tetrapyrrole signaling is poorly understood, there is compelling evidence that this signaling is significant. Specifically, this signaling appears to affect the accumulation of starch and may promote abiotic stress tolerance. Tetrapyrrole-dependent plastid-to-nucleus signaling interacts with a distinct plastid-to-nucleus signaling mechanism that depends

Edited by:

Shu Yuan,

Sichuan Agricultural University, China

Reviewed by:

Rongcheng Lin,

Chinese Academy of Sciences, China Tatsuru Masuda,

University of Tokyo, Japan

*Correspondence:

Robert M. Larkin

larkin@mail.hzau.edu.cn

Specialty section: This article was submitted to

Plant Physiology,

a section of the journal

Frontiers in Plant Science

Received: 30 July 2016 Accepted: 07 October 2016 Published: 19 October 2016

Citation: Larkin RM (2016) Tetrapyrrole Signaling in Plants.

Front. Plant Sci. 7:1586. doi: 10.3389/fpls.2016.01586 on GENOMES UNCUOPLED1 (GUN1). GUN1 contributes to a variety of processes, such as chloroplast biogenesis, the circadian rhythm, abiotic stress tolerance, and development. Thus, the contribution of tetrapyrrole signaling to plant function is potentially broader than we currently appreciate. In this review, I discuss these aspects of tetrapyrrole signaling.

Keywords: tetrapyrrole, porphyrin, heme, Mg-protoporphyrin IX, plastid signaling, plastid, chloroplast, chlorophyll

\section{INTRODUCTION}

Tetrapyrroles contribute a number of functions to processes that are critical for the viability of diverse organisms including essential functions to the light reactions photosynthesis, which underpins life on earth. Tetrapyrroles contain four pyrroles - aromatic rings containing four carbon atoms and one nitrogen atom-often linked together with methine groups, in linear (e.g., bilins) or cyclic arrangements (e.g., porphyrins). Porphyrins chelate metal ions, such as cobalt, iron, or magnesium ions. These structural features along with a variety of ring substituents contribute to the diverse functions of tetrapyrroles (Tanaka and Tanaka, 2007; Tanaka et al., 2011; Brzezowski et al., 2015; Figure 1).

In plants and algae, tetrapyrroles are synthesized ultimately from glutamate by a branched biosynthetic pathway localized entirely within plastids. The end products of this branched pathway are siroheme, heme, phytochromobilin, chlorophyll $a$, and chlorophyll $b$ (Figure 2). These tetrapyrroles provide critical functions to important processes, such as light signaling, 


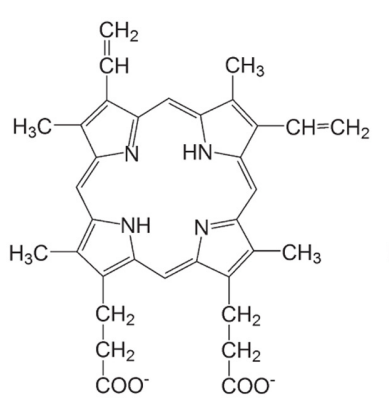

Protoporphyrin IX

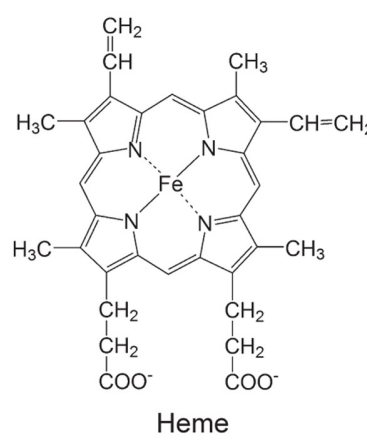

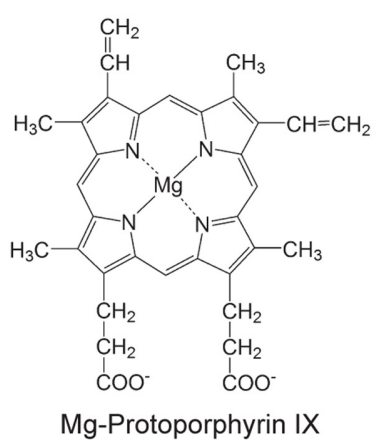

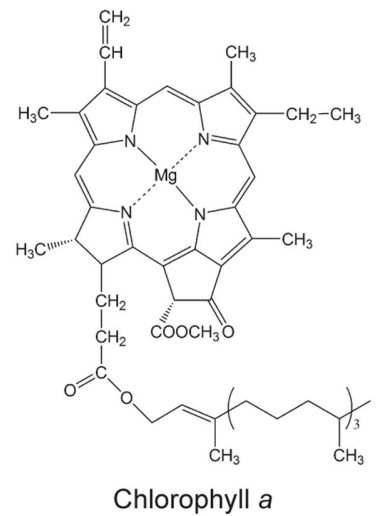<smiles>C=CC1=C(C)/C(=C\C2=N/C(=C\c3[nH]c(/C=C4\NC(=O)[C@H](C)\C4=C/C)c(C)c3CCC(=O)O)C(CCC(=O)O)=C2C)NC1=O</smiles>

3(E)-Phytochromobilin

FIGURE 1 | Selected tetrapyrroles from plants. The structures were adapted from Tanaka and Tanaka (2007).

the detoxification of reactive oxygen species, the assimilation of nitrate and sulfate, respiration, programmed cell death, and the light-harvesting reactions of photosynthesis. In vivo, many tetrapyrroles, such as porphyrins, are sequestered to prevent them from generating singlet oxygen $\left({ }^{1} \mathrm{O}_{2}\right)$, a toxic reactive oxygen species that causes stress and cell death. Electronically excited versions of porphyrins produce ${ }^{1} \mathrm{O}_{2}$ when they transfer energy to molecular oxygen (Tanaka and Tanaka, 2007; Tanaka et al., 2011; Brzezowski et al., 2015).

Considering the large number of critical functions performed by tetrapyrroles and the potentially lethal consequences of misregulating tetrapyrrole biosynthesis, it is not surprising that the levels of tetrapyrroles are strictly controlled (Tanaka and Tanaka, 2007; Tanaka et al., 2011; Brzezowski et al., 2015) and that tetrapyrroles affect signaling mechanisms that regulate gene expression in diverse organisms (Terry and Smith, 2013; Brzezowski et al., 2015; Zhang et al., 2015). Indeed, tetrapyrroles were some of the first molecules suggested to serve as plastid signals that regulate nuclear gene expression in both plants and algae (Susek and Chory, 1992). The idea that in addition to serving as the metabolic centers of plant cells, plastids serve as sensors that emit signals that regulate gene expression in the nucleus was first suggested almost forty years ago (Bradbeer et al., 1979). Since the early days of plastid-to-nucleus signaling research, researchers have studied the plastid regulation of photosynthesis-associated nuclear genes (PhANGs), especially the genes encoding the type I proteins of the major light harvesting complex of photosystem II (Lhcb1) (Susek and Chory, 1992; Gray et al., 2003). The Lhcb1 proteins accumulate in the thylakoid membranes where they bind chlorophylls, carotenoids, and serve as antennae for photosystem II (PSII) (Jansson et al., 1992). The expression of $L h c b$ genes is well studied. Additionally, when chloroplasts experience dysfunction, the expression of Lhcb genes is severely downregulated relative to other PhANGs (Susek and Chory, 1992).

Since the early days of plastid-to-nucleus signaling research, mutant alleles, and inhibitors that specifically block chloroplast biogenesis by a variety of distinct mechanisms were used to induce the plastid-to-nucleus signaling mechanisms that downregulate the expression of $L h c b$ genes and other PhANGs (Susek and Chory, 1992; Gray et al., 2003). Chloroplast biogenesis refers to the development of chloroplasts from non-photosynthetic proplastids. Chloroplast biogenesis occurs during germination and leaf development (Pogson et al., 2015). Subsequently, chloroplast division and a mechanism that establishes the size of the chloroplast compartment drive the proliferation of chloroplasts (Osteryoung and Pyke, 2014; Larkin et al., 2016). Experiments with inhibitors and mutant alleles that block chloroplast biogenesis indicated that the expression of PhANGs depends on chloroplast biogenesis and that the plastid-to-nucleus signaling that is activated by blocking chloroplast biogenesis typically downregulates the transcription of PhANGs (Susek and Chory, 1992; Gray et al., 2003). However, there is evidence for plastid signals using a posttranscriptional 


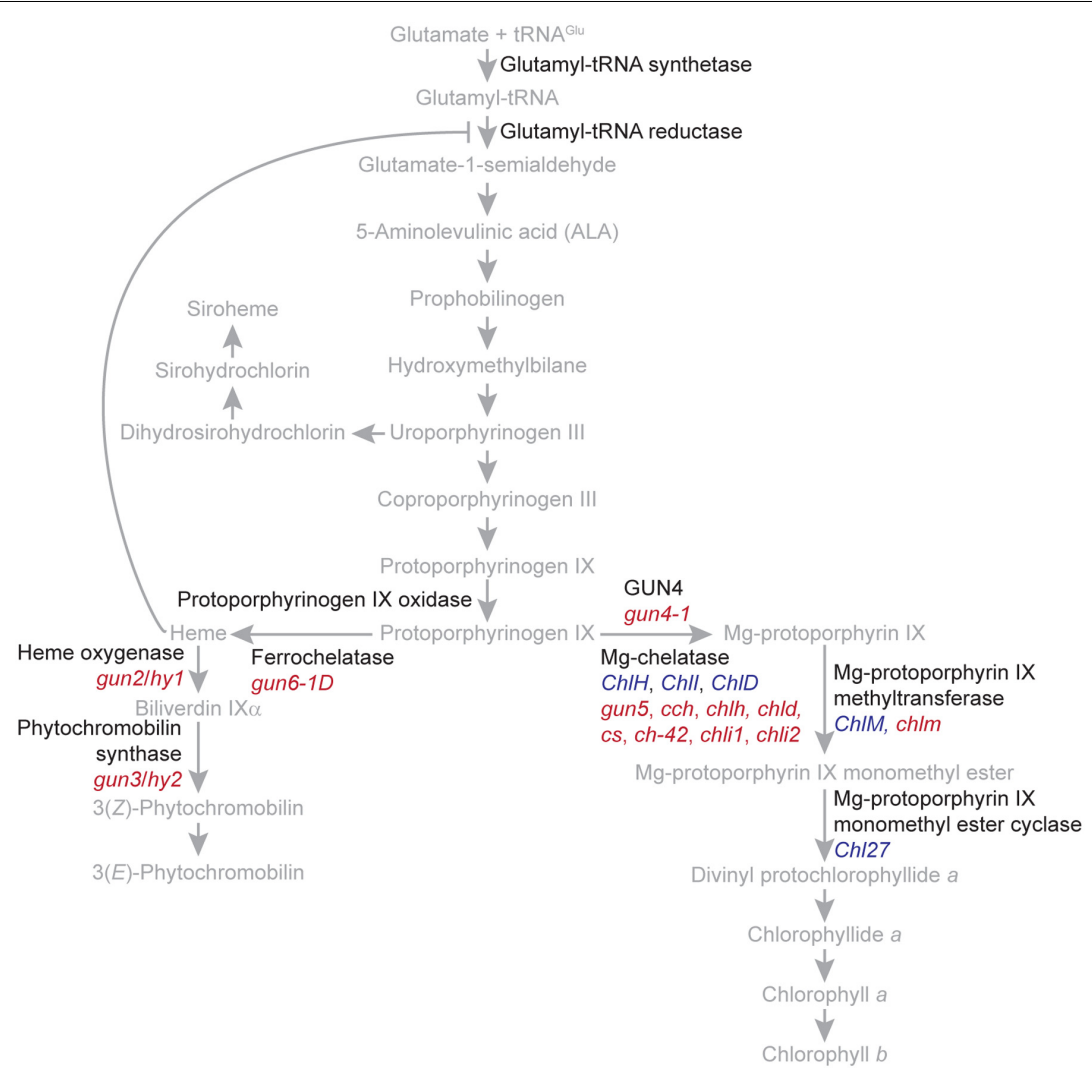

FIGURE 2 | The branched tetrapyrrole biosynthetic pathway of plants. The names of the precursors and end products of the branched tetrapyrrole biosynthetic pathway are indicated with gray. Enzyme catalyzed reactions are indicated with gray arrows. The feedback inhibition of glutamyl tRNA reductase by heme is indicated with a gray $T$ bar. The names of proteins, such as regulatory proteins and enzymes, are indicated with black. The tetrapyrrole biosynthetic pathway was adapted from Tanaka and Tanaka (2007) and Tanaka et al. (2011). Genes encoding enzymes or enzyme subunits are indicated with blue. Mutants discussed in the text are indicated with red. gun5, cch, and chlh are mutant alleles of ChIH/GUN5. ch/d is a mutant allele of ChID. cs, ch-42, and chli1 are mutant alleles of Chll1. chli2 is a mutant allele of Chll2.

mechanism to regulate the expression of a PhANG. The plastid regulated expression of PetE from pea requires an $\mathrm{mRNA}$ with an intact $5^{\prime}$ end and depends on translation in transgenic tobacco and transgenic Arabidopsis (Sullivan and Gray, 2002; Brown et al., 2005). During the early days of plastid-to-nucleus signaling research, a porphyrin was considered an attractive candidate for a plastid signal that regulates nuclear gene expression because at the time, heme was known to regulate nuclear gene expression in Saccharomyces cerevisiae (Forsburg and Guarente, 1989) and an inverse correlation was reported between the levels of the chlorophyll precursor Mg-protoporphyrin IX monomethyl ester (Mg-ProtoME) and the levels of the mRNAs that encode the Lhcb proteins and the small subunit of RuBisCO in Chlamydomonas reinhardtii (Johanningmeier and Howell, 1984).

Now, there is a larger body of evidence favoring the idea that porphyrins and bilins serve as plastid signals that regulate nuclear gene expression in both plants and algae (Terry and Smith, 2013; Brzezowski et al., 2015; Zhang et al., 2015). Additionally, we now know that there are a number of nonporphyrin plastid signals that activate distinct plastid-to-nucleus signaling mechanisms. We also know that plastid-to-nucleus signaling is not only a feedback mechanism that optimizes chloroplast function but that plastid-to-nucleus signaling affects extraplastidic processes (Larkin, 2014; Chan et al., 2016). In addition to affecting entire cells by regulating nuclear gene expression, plastid signals affect individual chloroplasts by promoting the degradation of individual chloroplasts that suffer from photooxidative stress, apparently by stimulating the ubiquitination of chloroplast proteins that are located on the cytosolic surface of chloroplasts (Woodson et al., 2015). Indeed, plastid-to-nucleus signaling mechanisms broadly contribute to plant function by serving as major regulators of plant cells.

Plastid-to-nucleus signaling research is frequently and extensively reviewed. Here, I review the data indicating that porphyrins regulate nuclear gene expression and the evidence for particular mechanisms of porphyrin-regulated gene expression in plants. I also review possible biological functions for porphyrin-dependent plastid-to-nucleus signaling and our understanding of the interactions between a porphyrin-dependent signaling mechanism with a signaling mechanism that does not appear to require porphyrins for its activation. 


\section{EARLY EXPERIMENTS RELEVANT TO TETRAPYRROLE SIGNALING IN PLANTS}

During the early days of plastid-to-nucleus signaling research, a number experiments were performed that tested whether porphyrins might contribute to the plastid regulation of nuclear gene expression in plants, but there were major technical obstacles. First, plants were thought to not take up porphyrins, such as the chlorophyll precursor Mg-ProtoME that was implicated as a plastid signal in C. reinhardtii (Johanningmeier and Howell, 1984), or similar porphyrins, such as Mg-protoporphyrin IX (Mg-Proto), and hemin. Second, the porphyrin precursors of chlorophyll, such as Mg-Proto and $\mathrm{Mg}$ ProtoME, are potent photosensitizers. Third, the model genes for studying plastid-to-nucleus signaling were PhANGs. The expression of PhANGs is strongly induced by qualities and quantities of light that would induce severe photooxidative stress if porphyrins were to accumulate and diffuse freely throughout plant cells.

Researchers overcame these technical barriers by feeding 5-aminolevulinic acid (ALA) to plants. ALA is the 5-carbon universal precursor of tetrapyrroles. ALA is hydrophilic and is readily taken up by plants. Exogenous ALA was demonstrated to flood the tetrapyrrole biosynthetic pathway with intermediates, induce increases in the levels of chlorophyll precursors (Granick, 1959; Gough, 1972; Mascia, 1978), and downregulate the expression of Lhcb genes in Lepidium sativum and Arabidopsis thaliana seedlings in non-photosensitizing conditions, such as after a non-photosensitizing pulse of red light, continuous illumination with non-photosensitizing far-red light, or the dark (Kittsteiner et al., 1991; Vinti et al., 2000). These data provide evidence that the accumulation of chlorophyll precursors, such as Mg-Proto or Mg-ProtoME, or that the accumulation of other plastid-derived tetrapyrroles regulate the expression of PhANGs in plants. Similar results were obtained after treating plants with inhibitors that induce increases in the levels of Mg-ProtoMe and Mg-Proto in non-photosensitizing conditions (Oster et al., 1996; La Rocca et al., 2001). More recently, ALA was suggested to mediate the effects of Mg-porphyrin metabolism on nuclear gene expression (Czarnecki et al., 2012)

\section{THE gun MUTANT SCREEN}

Developing mutant screens that specifically interrogate particular mechanisms is an objective approach for identifying completely unknown mechanisms. Thus, developing a specific mutant screen was an attractive approach for studying the plastid-tonucleus signaling that is activated when chloroplast biogenesis is blocked. Developing specific mutant screens remains an attractive approach for studying diverse plastid-to-nucleus signaling mechanisms because our current knowledge of plastidto-nucleus signaling mechanisms indicates that many plastid-tonucleus signaling mechanisms are not intuitively obvious (Chan et al., 2016). The first specific mutant screen developed that specifically interrogates plastid-to-nucleus signaling mechanisms was the genomes uncoupled (gun) mutant screen (Susek and
Chory, 1992; Susek et al., 1993). gun mutant screens use reporter genes to screen for Arabidopsis mutants that transcribe elevated levels of PhANGs, such as Lhcb genes, when chloroplast biogenesis is blocked with an herbicide treatment (Susek et al., 1993; Koussevitzky et al., 2007; Ruckle et al., 2007; Cottage et al., 2008; Woodson et al., 2011). gun alleles do not affect the expression of PhANGs in green seedlings containing wellfunctioning chloroplasts. gun alleles may attenuate the activity of negative regulators of PhANG expression, or gun alleles may induce the activity of positive regulators of PhANG expression (Susek et al., 1993; Mochizuki et al., 2001; Koussevitzky et al., 2007; Ruckle et al., 2007; Woodson et al., 2011). In addition to regulating the expression of PhANGs when chloroplast biogenesis is blocked, the plastid-to-nucleus signaling that depends on the GUN genes was shown to regulate the expression of PhANGs when chloroplast and etioplast function is somewhat attenuated (Koussevitzky et al., 2007; Sun et al., 2011; Woodson et al., 2013; Tadini et al., 2016).

gun mutant screens yielded a large number of mutant alleles of genes that encode a chloroplastic pentatricopeptide repeat protein named GUN1 (Susek et al., 1993; Koussevitzky et al., 2007; Cottage et al., 2008), a photoreceptor that is activated by blue light named cryptochrome1 (cry1) (Ruckle et al., 2007), and genes that contribute to tetrapyrrole metabolism (Mochizuki et al., 2001; Larkin et al., 2003; Adhikari et al., 2011). These data indicate that gun mutant screens specifically disrupt a few distinct mechanisms that downregulate the expression of $L h c b$ genes when chloroplast biogenesis is blocked and that when chloroplast biogenesis is blocked, specific mechanisms downregulate the expression of PhANGs. The specificity of the gun mutant screen was first demonstrated more than 20 years ago (Susek et al., 1993). The data from Susek et al. (1993) and the data from subsequent analyses of gun mutant screens (Mochizuki et al., 2001; Larkin et al., 2003; Koussevitzky et al., 2007; Ruckle et al., 2007; Cottage et al., 2008; Adhikari et al., 2011) conflict with a commonly promoted alternative interpretation that unnatural and complex metabolism downregulates the expression of PhANGs when chloroplast biogenesis is blocked (Pfannschmidt, 2010; Voigt et al., 2010; Barajas-López et al., 2012; Kindgren et al., 2012; Terry and Smith, 2013).

\section{GUN2, GUN3, GUN4, GUN5, AND GUN6}

The gun screen yielded several mutant alleles of genes that contribute to tetrapyrrole metabolism named GUN2, GUN3, GUN4, GUN5, and GUN6 (Figure 2). GUN2 encodes heme oxygenase. GUN3 encodes phytochromobilin synthase (Mochizuki et al., 2001). These enzymes contribute to the biosynthesis of phytochromobilin, the chromophore of phytochromes (Tanaka and Tanaka, 2007). gun2 and gun3 are allelic to $h y 1$ and $h y 2$, which disrupt photomorphogenesis (Koornneef et al., 1980). A hyl mutant was independently demonstrated to exhibit a gun phenotype (Vinti et al., 2000). GUN5 encodes the $140-\mathrm{kDa}$ subunit of Mg-chelatase (Mochizuki et al., 2001). GUN4 encodes an activator of Mg-chelatase that binds the $140-\mathrm{kDa}$ subunit of Mg-chelatase and the 
porphyrin substrate and product of $\mathrm{Mg}$-chelatase (Larkin et al., 2003; Davison et al., 2005; Verdecia et al., 2005; Adhikari et al., 2009). GUN4 is only found in organisms that perform oxygenic photosynthesis (Larkin et al., 2003). GUN4 was demonstrated to shield porphyrins from collisions with molecular oxygen that produce ${ }^{1} \mathrm{O}_{2}$ (Brzezowski et al., 2014), as was suggested previously (Larkin et al., 2003; Verdecia et al., 2005). However, the subsequent finding that porphyrin-binding pocket of GUN4 is partially opened (Chen et al., 2015) and that in vitro, porphyrins produce more ${ }^{1} \mathrm{O}_{2}$ when they are bound to GUN4 (Tarahi Tabrizi et al., 2016) indicates that GUN4 requires additional factors to shield porphyrins from collisions with molecular oxygen. Thus, GUN4 and GUN5 divert protoporphyrin IX from heme biosynthesis to chlorophyll biosynthesis by inserting a magnesium ion into protoporphyrin IX, yielding Mg-Proto. GUN6 encodes ferrochelatase1 (FC1), which synthesizes heme from protoporphyrin IX (Woodson et al., 2011). The cloning of so many mutant alleles that disrupt tetrapyrrole metabolism indicates that gun mutant screens specifically disrupt a plastid-to-nucleus signaling mechanism that is regulated by tetrapyrrole metabolism. Consistent with these data, porphyrins serve as plastid signals in diverse organisms. Null alleles of GUN4 affected nuclear gene expression in C. reinhardtii-even in dark-grown cultures (Formighieri et al., 2012), both Mg-Proto and heme were reported to serve as plastid signals in algae (Beck, 2005; von Gromoff et al., 2008; Voß et al., 2011; Tanaka and Hanaoka, 2013), and heme was demonstrated to regulate various signaling mechanisms in diverse organisms, such as bacteria, red algae, yeast, and animals (Tanaka and Hanaoka, 2013; Terry and Smith, 2013; Kobayashi et al., 2016).

In the gun mutant screen, norflurazon is typically used to block chloroplast biogenesis. This herbicide specifically inhibits phytoene desaturase, which plays an essential role in carotenoid biosynthesis by catalyzing two sequential dehydrogenation reactions on phytoene, yielding $\zeta$-carotene. Thus, norflurazon treatments inhibit the accumulation of carotenoids. Carotenoids are essential for quenching triplet chlorophyll and ${ }^{1} \mathrm{O}_{2}$ in green tissues (Susek and Chory, 1992; Gray et al., 2003; Nisar et al., 2015). Thus, when green leaves are treated with norflurazon, ${ }^{1} \mathrm{O}_{2}$ accumulates and leaves suffer from severe photooxidative stress (Kim and Apel, 2013). However, in the gun mutant screen, Arabidopsis seeds are germinated on a growth medium containing norflurazon and subsequently, seedlings are grown for several days on the same medium until their gene expression phenotypes are scored. In these conditions, chlorophyll does not accumulate, the thylakoid membranes do not develop, chloroplast biogenesis appears arrested at an early stage, and plastids resemble proplastids (Susek et al., 1993). Norflurazon was proposed to block chloroplast biogenesis by causing photooxidative stress during the conversion of proplastids to chloroplasts (Susek and Chory, 1992; Gray et al., 2003). This model assumes that when seedlings are germinated on a medium containing norflurazon-during the biogenesis of chloroplasts from non-photosynthetic proplastids-free chlorophyll or free chlorophyll precursors accumulate without the concomitant accumulation of carotenoids. This model assumes that these chlorophylls or chlorophyll precursors drive the accumulation of ${ }^{1} \mathrm{O}_{2}$ and that the resulting photooxidative stress blocks chloroplast biogenesis (Susek and Chory, 1992; Gray et al., 2003).

A variety of independent experiments-including experiments with ${ }^{1} \mathrm{O}_{2}$-detecting dyes and ${ }^{1} \mathrm{O}_{2}$-regulated genes-unambiguously indicated that ${ }^{1} \mathrm{O}_{2}$ accumulates in green Arabidopsis leaves treated with norflurazon (Kim and Apel, 2013). In stark contrast, the same methods for detecting ${ }^{1} \mathrm{O}_{2}$ indicated that ${ }^{1} \mathrm{O}_{2}$ does not appear to accumulate when Arabidopsis seeds are germinated on a medium containing norflurazon (Kim and Apel, 2013). One interpretation of these data is that norflurazon might use a different mechanism to photobleach green leaves and to block chloroplast biogenesis during germination. For instance, the coordinated downregulation of both carotenoid and chlorophyll biosynthesis was suggested to block chloroplast biogenesis by preventing the biogenesis of the thylakoid membranes, without inducing photooxidative stress (Kim and Apel, 2013). Indeed, neither chlorophyll nor its precursors accumulate when Arabidopsis seedlings are germinated on a medium containing norflurazon (Mochizuki et al., 2008; Moulin et al., 2008).

An alternative interpretation is that a transient production of ROS blocks chloroplast biogenesis when Arabidopsis seeds are germinated on a medium containing norflurazon and because of the transient nature of this increase in the levels of ROS, these ROS are impractical or impossible to detect (Mochizuki et al., 2010; Kim and Apel, 2013). Consistent with this interpretation, when seeds were germinated on a medium containing norflurazon, the expression of one ${ }^{1} \mathrm{O}_{2}$-inducible gene was higher after 3 days than after 5 days of growth (Kim and Apel, 2013). However, changes in the expression of more than one ${ }^{1} \mathrm{O}_{2}$-inducible gene are required to provide evidence for elevated levels of ${ }^{1} \mathrm{O}_{2}$ because genes are typically regulated by more than one signal. Nonetheless, light influences the ability of norflurazon to downregulate the expression of nuclear genes that are regulated by plastid signals, which provides more evidence for a mechanism that depends on photooxidative stress (Susek and Chory, 1992). For this reason, norflurazontreated seedlings are typically grown in bright white light (e.g., at least $100 \mu \mathrm{mol} \mathrm{m} \mathrm{m}^{-2} \mathrm{~s}^{-1}$ ) to test for gun phenotypes. However, norflurazon appeared to block chloroplast biogenesis when Arabidopsis seedlings were grown in $10 \mu \mathrm{mol} \mathrm{m} \mathrm{m}^{-2} \mathrm{~s}^{-1}$ white light (Voigt et al., 2010). The impact of chloroplast function on light signaling (Lepistö and Rintamäki, 2012; Larkin, 2014) may at least partially explain the effect of the fluence rate on norflurazon treatments. Light signaling generally induces the expression of PhANGs in green seedlings but when chloroplast biogenesis is blocked, light signaling becomes a negative regulator of Lhcb expression (Ruckle et al., 2007 and Figure 1 from Ruckle et al., 2012) and severely attenuates the expression of many other PhANGs (Ruckle et al., 2012). This conversion of light signaling from a positive to a negative regulator of $L h c b$ genes while only attenuating the light-induced expression of other PhANGs may explain why blocking chloroplast biogenesis with norflurazon treatments downregulates the expression of Lhcb genes more than other PhANGs (Susek and Chory, 1992). 
A number of authors have promoted the idea that the gun mutants with deficiencies in tetrapyrrole metabolism are partially resistant to norflurazon without discussing the data that conflict with this interpretation. Their interpretation is that norflurazon is less effective in mutants that are partially chlorophyll deficient because lower levels of chlorophyll biosynthesis reduce the amount of ROS produced during chloroplast biogenesis in norflurazon-treated seedlings. Therefore, in these gun mutants, they propose that the norflurazon-induced inhibition of chloroplast biogenesis is not as severe as in wild-type seedlings. Consequentially, they propose that PhANGs are expressed at higher levels in these gun mutants than in wild type because PhANG expression is correlated with chloroplast biogenesis (Mochizuki et al., 2008; Moulin et al., 2008; Kleine et al., 2009; Galvez-Valdivieso and Mullineaux, 2010; Mochizuki et al., 2010; Armbruster et al., 2011; Jarvis and López-Juez, 2013; Kleine and Leister, 2013; Terry and Smith, 2013; Bobik and Burch-Smith, 2015).

The first indication that the chlorophyll-deficient gun mutants are not resistant to norflurazon was the lack of correlation between chlorophyll-deficient phenotypes and the gun phenotypes, which was apparent after the characterization of a number of tetrapyrrole biosynthesis mutants from Arabidopsis. For instance, the $40-\mathrm{kDa}$ subunit of Mg-chelatase is encoded by two genes named ChlI1 and ChlI2. A ChlI1 mutant named ch42-1 was reported to accumulate $11 \%$ of the chlorophyll found in wild type or to not accumulate chlorophyll (Mochizuki et al., 2001; Rissler et al., 2002). When ChlI2 was knocked out, chlorophyll levels were significantly less but similar to wild type (Kobayashi et al., 2008). Thus, CHLI1 encodes the predominant isoform of this Mg-chelatase subunit. $c s$ is a leaky loss-of-function allele of ChlI1 (Koncz et al., 1990). cs and gun4-1 accumulated less than $40 \%$ of the chlorophyll found in wild type. cch and gun 5 are GUN5 mutants that accumulated approximately 35 and $70 \%$ of the chlorophyll found in wild type (Mochizuki et al., 2001). In contrast to cch, gun4-1, and gun5, neither cs nor ch42-1 expressed higher levels of Lhcb1 than wild type when chloroplast biogenesis was blocked with a norflurazon treatment (i.e., cch, gun4-1, and gun5 are gun mutants. cs and ch42-1 are not gun mutants) (Mochizuki et al., 2001). Additionally, the gun phenotype appeared significantly attenuated in the completely albino hy 1 gun 5 double mutant relative to the partially chlorophyll deficient gun5 and hy1 (i.e., gun2) mutants (Vinti et al., 2000). Thus, there is no correlation between chlorophyll deficient phenotypes and gun phenotypes.

T-DNA insertion alleles of the genes encoding the subunits of Mg-chelatase that induce severe chlorophyll deficiencies or albinism also induce gun phenotypes (Huang and Li, 2009). In contrast to previous work that used northern blots to test for gun phenotypes (Vinti et al., 2000; Mochizuki et al., 2001), Huang and Li (2009) quantified the expression of Lhcb1 genes using qRT-PCR. They found an approximately twofold increase in Lhcb1 expression in the severely chlorophyll-deficient chli1 mutants and 4.5- to 8-fold increases in the albino chld and chlh mutants, which are null alleles of the genes encoding the 80 - and $140-\mathrm{kDa}$ subunits of $\mathrm{Mg}$-chelatase, respectively. The finding that CHLI1 mutants were either not gun mutants or were subtle gun mutants depending on whether $L h c b 1$ expression was quantified by northern blotting or by qRT-PCR and that $\mathrm{CHLH}$ mutants exhibited robust gun phenotypes regardless of the method used for quantifying $L h c b 1$ expression are essentially the same results.

Additional data conflict with the idea that partial resistance to norflurazon explains the gun phenotypes of the gun mutants that have defects in tetrapyrrole metabolism. (1) Attempts to show that norflurazon-treated gun4-1 and gun 5 accumulate less reactive oxygen species (ROS) than norflurazon-treated wild type by quantifying ROS-responsive gene expression, staining with ROS responsive dyes, and by treating plants with ROS scavengers were not successful (Strand et al., 2003; Voigt et al., 2010; Zhang et al., 2011a; Kim and Apel, 2013). (2) No gun mutants were obtained from screens for norflurazon resistant mutants (i.e., the happy on norflurazon mutants) (Saini et al., 2011). (3) gun4-1 and gun5 were not resistant to low concentrations of norflurazon, in contrast to the happy on norflurazon mutants (Saini et al., 2011). (4) Blocking chloroplast biogenesis with a norflurazon treatment activated plastid-to-nucleus signaling mechanisms that are distinct from the ${ }^{1} \mathrm{O}_{2}$-dependent plastidto-nucleus signaling mechanisms that were activated when green seedlings were treated with norflurazon and consequentially the levels of chloroplastic ${ }^{1} \mathrm{O}_{2}$ were increased (Kim and Apel, 2013). (5) High-fluence-rate light and a ROS-inducing methyl viologen treatment required different promoter elements than norflurazon treatments that blocked chloroplast biogenesis to downregulate the expression of the $L h c b 1^{*} 2$ promoter from Nicotiana plumbaginifolia (Staneloni et al., 2008). (6) Null alleles of sigma factor2 (sig2) reduced chlorophyll levels to $20 \%$ of the chlorophyll found in wild type. Thus, in contrast to norflurazon treatments, sig2 attenuates but does not block chloroplast biogenesis. sig2 mutants expressed lower levels of PhANGs than wild type. Etiolated gun5 sig2 expressed higher levels Lhcb2.2 and other PhANGs than etiolated sig2 (Woodson et al., 2013). Thus, perturbing tetrapyrrole metabolism affected nuclear gene expression in dark-grown Arabidopsis seedlings that were not treated with norflurazon. (7) Tetrapyrrole metabolism regulated starch-related nuclear gene expression in Bright Yellow-2 (BY2) cells derived from tobacco, which lack photosynthetic plastids (Enami et al., 2011). (8) In C. reinhardtii, mutant alleles of GUN4 and ChlM-the gene encoding Mg-protoporphyrin IX methyltransferase-upregulated the expression of genes that encode the LHC proteins and genes that contribute to tetrapyrrole metabolism in cells that were not treated with norflurazon (Meinecke et al., 2010; Formighieri et al., 2012; Brzezowski et al., 2014). (9) Phytoene desaturase was inhibited in norflurazon-treated gun mutants with deficiencies in tetrapyrrole metabolism, as a class. Thus, alternative mechanisms of partial resistance do not explain the phenotypes of these gun mutants (Voigt et al., 2010).

The idea that no evidence of partial resistance to norflurazon was obtained in so many independent experiments because during chloroplast biogenesis, the norflurazon-induced increase in ROS is transient or localized (Mochizuki et al., 2010; Kim and Apel, 2013) is difficult to reconcile with the data. This interpretation is especially difficult to reconcile with the findings that there was no correlation between the chlorophyll-deficient 
phenotypes and the gun phenotypes (Vinti et al., 2000; Mochizuki et al., 2001), etiolated gun5 sig2 expressed higher levels of several PhANGs than etiolated sig2 (Woodson et al., 2013), and neither gun4-1 nor gun5 were resistant to low concentrations of norflurazon-in contrast to norflurazon-resistant mutants (Saini et al., 2011). If norflurazon treatments block chloroplast biogenesis in Arabidopsis seedlings by inducing photooxidative stress, (1) chloroplast biogenesis in Arabidopsis seedlings would need to exhibit such extreme sensitivity to this photooxidative stress that the perturbations in the tetrapyrrole biosynthetic pathway exhibited by gun2, gun3, gun4-1, gun 5, and gun6-1D would not affect the photobleaching activity of norflurazon and (2) the perturbations in tetrapyrrole metabolism induced by gun 5 would need to affect a distinct mechanism to affect the expression of PhANGs in the dark.

\section{THE MECHANISM OF TETRAPYRROLE SIGNALING IN PLANTS: CHLOROPHYLL BIOSYNTHETIC ENZYMES AND CHLOROPHYLL PRECURSORS}

The mutant alleles of GUN2/HY1, GUN3/HY2, GUN4, and GUN5 that were isolated from gun mutant screens are loss-of-function alleles (Mochizuki et al., 2001; Larkin et al., 2003; Adhikari et al., 2011). In contrast, the mutant allele of GUN6 that was isolated by Woodson et al. (2011) is a gain-of-function allele. These mutant alleles reduce the flux through the chlorophyll branch by two distinct mechanisms: (1) directly inhibiting chlorophyll biosynthesis or (2) promoting the accumulation of heme. Heme inhibits chlorophyll biosynthesis by attenuating the activity of glutamyl tRNA reductase. These mutant alleles may also induce increases in the levels of heme by diverting protoporphyrin IX from the chlorophyll branch to the heme branch, increasing ferrochelatase activity, or by attenuating the activities of heme oxygenase and phytochromobilin synthase (Vinti et al., 2000; Mochizuki et al., 2001; Larkin et al., 2003; Woodson et al., 2011).

Based on the finding that chlorophyll biosynthesis is attenuated in gun4, gun5, cch, cs, and ch42-1, and that in contrast to gun4, gun5, and cch, neither cs nor the ch421 are gun mutants, tetrapyrrole-dependent plastid-to-nucleus signaling was proposed to not depend on the accumulation of a particular chlorophyll precursor, such as Mg-Proto or MgProtoME. Instead, GUN5 was proposed to participate in plastidto-nucleus signaling (Mochizuki et al., 2001). The turnover of a porphyrin, such as Mg-Proto, or changes in the subchloroplastic localization of GUN4, GUN5, or a GUN4-GUN5 complex associated or not associated with a porphyrin ligand was suggested affect plastid-to-nucleus signaling (Mochizuki et al., 2001, 2008, 2010; Larkin et al., 2003; Sun et al., 2011). Consistent with these ideas, a number of proteins perform more than one function, such as distinct functions in metabolism and signaling (Boukouris et al., 2016). Indeed, the ortholog of GUN5 from Synechocystis sp. PCC 6803 serves as both a Mg-chelatase subunit and as an anti-sigma factor (Osanai et al., 2009).
The finding that porphyrin binding increases the affinity of GUN4 and GUN5 for chloroplast membranes (Adhikari et al., 2009, 2011) is also consistent with these ideas because the chloroplast envelope membrane may serve as an important subchloroplastic location for communication between chloroplasts and extrachloroplastic compartments.

Subsequently, the levels of Mg-Proto were reported to accumulate when chloroplast biogenesis was blocked with a norflurazon treatment, and Mg-Proto was reported to accumulate to lower levels in gun2 and gun5 (Strand et al., 2003). Data from a number of additional experiments performed by Strand et al. (2003) supported the idea that the accumulation of Mg-Proto is a plastid signal that downregulates PhANG expression, including an experiment showing that exogenous Mg-Proto downregulated the expression of $L h c b$ genes in protoplasts and that exogenous hemin did not affect the expression of $L h c b$ genes in protoplasts. The data from Strand et al. (2003) conflict with the data reported by Mochizuki et al. (2001) and provide evidence that the chlorophyll-deficient gun mutants affect plastid-to-nucleus signaling by reducing the levels of Mg-Proto. Subsequently, other work provided more evidence that the accumulation of a particular Mg-porphyrin might serve as a plastid signal. A barley mutant deficient in the Mg-ProtoME cyclase accumulated Mg-ProtoME and did not exhibit a gun phenotype (Gadjieva et al., 2005). An Arabidopsis mutant deficient in the Mg-ProtoME cyclase accumulated MgProto and did not exhibit a gun phenotype (Ankele et al., 2007). An Arabidopsis mutant deficient in Mg-Proto methyltransferase accumulated Mg-Proto and did not exhibit a gun phenotype (Pontier et al., 2007). Based on these data, these authors suggested that the accumulation of either Mg-Proto (Ankele et al., 2007; Pontier et al., 2007) or Mg-ProtoME (Gadjieva et al., 2005) might serve as a plastid signal that downregulates the expression of PhANGs. Modulating the levels of Mg-Proto methyltransferase with sense or antisense expression in tobacco was reported to affect PhANG expression, which is also consistent with $\mathrm{Mg}$ porphyrins serving as a plastid signal (Alawady and Grimm, 2005). Using confocal laser scanning spectroscopy, Mg-Proto or Mg-ProtoME were detected in the cytosol of ALA fed Arabidopsis plants that were treated or not treated with norflurazon (Ankele et al., 2007; Zhang et al., 2011a). In one instance, Mg-Proto or Mg-ProtoME were detected in the cytosol of cells from plants that were not fed with ALA (Zhang et al., 2011a). Thus, plastids are capable of exporting Mg-Proto or Mg-ProtoME. These data are consistent with plastids exporting Mg-Proto or Mg-ProtoME and with Mg-Proto or Mg-ProtoME activating a signaling mechanism in the cytosol that regulates the expression of PhANGs.

As discussed above, researchers commonly elevated the endogenous levels of porphyrins with exogenous ALA because plant roots were thought to not take up porphyrins, such as Mg-Proto. More recently, Arabidopsis plants were reported to take up Mg-Proto through their roots when they were simply watered with solutions containing Mg-Proto (Zhang et al., 2011a; Kindgren et al., 2012; Barajas-López et al., 2013). An increase in the efficiency of Mg-Proto and hemin feeding was reported by first vacuum infiltrating seedlings prior to root feeding (Zhang et al., 2013). In one instance, feeding Mg-Proto to plants 
through their roots was reported to induce an increase in the areal levels of Mg-Proto (Kindgren et al., 2012). Exogenous Mg-Proto, Mg-ProtoME, or hemin doubled the cellular RNA content in Arabidopsis plants (Zhang et al., 2011b). Feeding both Mg-Proto and hemin to plants downregulated the expression of $L h c b$ and RbcS genes (Zhang et al., 2011a, 2013; Kindgren et al., 2012; Barajas-López et al., 2013). This reduced expression of PhANGs that was induced by exogenous porphyrins was attenuated in gun1, long hypocotyl5 (hy5), aba-insentive4 (abi4), and phytochrome-associated protein phosphatase 5 (papp5) (Zhang et al., 2011a; Kindgren et al., 2012; Barajas-López et al., 2013). These findings are consistent with tetrapyrrole metabolism affecting plastid-to-nucleus signaling upstream of the chloroplastic pentatricopeptide protein GUN1 and with PAPP5 and the nuclear transcription factors HY5 and ABI4 contributing to this mechanism. Exogenous porphyrins affecting GUN1dependent plastid-to-nucleus signaling is consistent with purified chloroplasts importing porphyrins in vitro (Adhikari et al., 2009, 2011). Feeding Mg-Proto and hemin to plants was also reported to affect the expression of genes encoding transcription factors, such as CAAT binding proteins (CBP) and the C-REPEAT BINDING FACTORS (CBF) (Zhang et al., 2013; Norén et al., 2016). In apparent conflict with these data collected from intact Arabidopsis plants, Strand et al. (2003) reported that exogenous hemin did not affect the expression of Lhcb in Arabidopsis protoplasts.

In stark contrast, three different laboratories demonstrated that Mg-Proto did not accumulate in barley and Arabidopsis when chloroplast biogenesis was blocked with norflurazon treatments (Gadjieva et al., 2005; Mochizuki et al., 2008; Moulin et al., 2008). Additionally, the norflurazon-treated chlm gun4 and the chlm gun5 double mutants accumulated elevated levels of Mg-Proto relative to gun4, gun5, and wild type but exhibited the same gun phenotypes as gun4 and gun5 when chloroplast biogenesis was blocked with a norflurazon treatment (Mochizuki et al., 2008). Exogenous ALA induced an approximately 200-fold increase in the levels of $\mathrm{Mg}$-Proto and Mg-ProtoME in etiolated Arabidopsis seedlings but had no impact on the subsequent far-red light-induced expression of Lhcb1 (Mochizuki et al., 2008). Elevated levels of Mg-Proto in transgenic rice plants that overexpress Myxococcus xanthus protoporphyrinogen IX oxidase did not reduced the levels of $L h c b$ expression relative to wild type (Phung et al., 2011). Moulin et al. (2008) suggested that the difference in the levels of Mg-Proto in norflurazon-treated seedlings reported by Strand et al. (2003) and Moulin et al. (2008) resulted from Strand et al. (2003) misidentifying a contaminating substance as Mg-Proto. Alternatively, a transient accumulation of Mg-Proto was suggested to explain this discrepancy (Kindgren et al., 2011, 2012). Indeed, transient accumulations of Mg-Proto were reported when plants were grown in photoperiodic light (Pöpperl et al., 1998; Papenbrock et al., 1999; Norén et al., 2016) or when chloroplasts experience photooxidative stress (Aarti et al., 2006; Kindgren et al., 2011, 2012; Zhang et al., 2011a). The problem with the proposal that a transient accumulation of Mg-Proto explains this discrepancy is that the growth conditions used to detect the gun phenotype do not fluctuate (i.e., constant temperature and continuous light of a constant fluence rate) to eliminate the possibility of the circadian regulation of PhANG expression obscuring the plastid regulation of PhANG expression (Susek et al., 1993). Indeed, transient peaks of Mg-Proto were not observed in plants grown in continuous light (Pöpperl et al., 1998; Papenbrock et al., 1999; Norén et al., 2016).

An alternative to the idea that a sustained accumulation of Mg-Proto in the chloroplast regulates gene expression in the nucleus is that a transient increase in the levels of $\mathrm{Mg}$-Proto might regulate gene expression in the nucleus. Indeed, transient changes in the levels of Mg-Proto and hemin affect plastid-tonucleus signaling in algae (Kropat et al., 2000; von Gromoff et al., 2008; Kobayashi et al., 2009; Voß et al., 2011). In 3-week-old green Arabidopsis plants, a transient increase in the levels of MgProto and Mg-ProtoME occurred $48 \mathrm{~h}$ after an oxidative-stress inducing norflurazon treatment (Zhang et al., 2011a). Similarly, oxidative stress resulting from a methyl viologen treatment induced a rise in the levels of $\mathrm{Mg}$-Proto and $\mathrm{Mg}$-ProtoME in cucumber and Arabidopsis (Aarti et al., 2006; Kindgren et al., 2011, 2012). Both treatments appeared to inhibit the activity of the Mg-ProtoME cyclase (Aarti et al., 2006; Zhang et al., 2011a; Kindgren et al., 2011, 2012). These herbicide treatments downregulated the expression of Lhcb genes (Zhang et al., 2011a; Kindgren et al., 2011, 2012). Zhang et al. (2011a) provided evidence that hydrogen peroxide and superoxide might not downregulate the expression of $L h c b$ genes by acting downstream of GUN5. In contrast, Kindgren et al. (2011, 2012) did not account for potential ROS effects on the expression of $L h c b$ genes. Zhang et al. (2011a) concluded that norflurazon treatments induce a transient increase in the levels of Mg-Proto in 3week-old plants and based on their data in 3-week-old plants inferred that in the gun mutant screen, norflurazon might induce a transient increase in the levels of Mg-Proto that leads to the long-term down-regulation of Lhcb expression. Kim and Apel (2013) performed an experiment with 5-day-old Arabidopsis seedlings that was similar to the experiment performed by Zhang et al. (2011a) with 3-week-old plants. Five-day-old seedlings were treated with norflurazon for $12 \mathrm{~h}$ in the dark and then returned to continuous light. This treatment induced increases in the levels of superoxide anion radical, hydrogen peroxide, ${ }^{1} \mathrm{O}_{2}$, and ${ }^{1} \mathrm{O}_{2}$ dependent plastid-to-nucleus signaling (Kim and Apel, 2013) but had no effect on the expression of Lhcb genes $24 \mathrm{~h}$ after the norflurazon-treated seedlings were transferred from the dark to the light. The different ages or the different methods of treating Arabidopsis plants with norflurazon may explain why Zhang et al. (2011a) observed a decrease in Lhcb expression $24 \mathrm{~h}$ after transferring norflurazon treated plants to bright light and Kim and Apel (2013) did not.

In contrast, Schlicke et al. (2014) induced a transient increase in Mg-porphyrin levels without first inducing photooxidative stress. They downregulated the expression of the genes encoding the $140-\mathrm{kDa}$ subunit of $\mathrm{Mg}$-chelatase $(\mathrm{ChlH}), \mathrm{Mg}$-Proto methyltransferase (ChlM), and Mg-ProtoME cyclase (Chl27) in 10-day-old Arabidopsis seedlings with an inducible RNAi system. Within $24 \mathrm{~h}, \mathrm{Mg}$-ProtoME accumulated-to higher levels than observed by Zhang et al. (2011a) - in the Chl27 RNAi lines. It is not clear whether the approach of Zhang et al. (2011a) or Schlicke et al. (2014) induced larger increases in the levels of 
Mg-Proto. In the Chl27 RNAi lines-24 h after suppression was induced-there were no macroscopic changes in the plants and no significant differences in the expression of PhANGs or ROSregulated genes. Four days of downregulated Chl27expression induced chlorophyll deficiencies, necrosis, and downregulated PhANG expression. Plastid-to-nucleus signaling was proposed to not cause the changes in gene expression that were observed $24 \mathrm{~h}$ after the RNAi-induced suppression of ChlH, ChlM, and Chl27 (Schlicke et al., 2014).

When plants are grown in photoperiodic light, transient increases in the levels of Mg-Proto and Mg-ProtoME routinely occur-apparently without causing photooxidative stress. For example, at dawn in tobacco and barley, Mg-Proto accumulated 30 (barely) and 60-fold (tobacco). Mg-ProtoME accumulated 100 (tobacco) and 200-fold (barley). This accumulation occurred within $30 \mathrm{~min}$ (tobacco) and $60 \mathrm{~min}$ (barley) and declined within another hour (Pöpperl et al., 1998). An independent study of the accumulation of $\mathrm{Mg}$-Proto and $\mathrm{Mg}$-ProtoME in tobacco in photoperiodic light appeared to miss the first striking peaks of Mg-Proto and Mg-ProtoME detected by Pöpperl et al. (1998) but appeared to detect the subsequent broad peak of $\mathrm{Mg}$-Proto and $\mathrm{Mg}$-ProtoME accumulation that occurred throughout the remainder of the light period (Papenbrock et al., 1999) that was also reported by Pöpperl et al. (1998). A similar broad peak of Mg-Proto and Mg-ProtoME accumulation was reported in Arabidopsis grown in photoperiodic light (Norén et al., 2016). In tobacco, increases in the expression of Lhcb genes preceded dawn, only increased for $6 \mathrm{~h}$, and began declining 8 h after dawn (Paulsen and Bogorad, 1988), several hours after the transient and striking increases in the levels of $\mathrm{Mg}$ Proto and Mg-ProtoME (Pöpperl et al., 1998). Thus, in tobacco, there is no correlation between the diurnal accumulation of these Mg-porphyrins (Pöpperl et al., 1998; Papenbrock et al., 1999) and the levels of Lhcb mRNA (Paulsen and Bogorad, 1988).

An attempt to identify the cytosolic receptor for Mg-Proto in plants revealed that a large number of different cytosolic proteins bound to an affinity column constructed by linking Mg-Proto to Affi-Gel 102 (Kindgren et al., 2011). Several different types of heat shock proteins bound to this column (Kindgren et al., 2011). The affinities of these heat shock proteins for Mg-Proto were not quantified. Although the finding that the HEAT SHOCK PROTEIN 90 (HSP90) bound to a Mg-Proto-affinity column and the finding that $20 \mu \mathrm{M} \mathrm{Mg-Proto}$ induced a $16 \%$ reduction in the ATPase activity of HSP90 provide evidence that HSP90s bind Mg-Proto (Kindgren et al., 2011, 2012), these data do not provide evidence for the high-affinity binding expected for a receptor.

Based on precedents for heat shock proteins contributing to heme signaling in yeast and a previously demonstrated connection between HY5 and both plastid-to-nucleus signaling and Lhcb expression, Kindgren et al. (2012) provided evidence that both HSP90 and HY5 contribute to the Mg-Proto-regulated expression of $L h c b$ genes. RNAi lines that reduced the expression of all HSP90.1-HSP90.4 genes exhibited lower levels of PhANG expression relative to wild type, but only norflurazon- or methyl viologen-treated lines were tested (Kindgren et al., 2012). Although gun mutants exhibit defects in PhANG expression when chloroplasts experience dysfunction, gun mutants do not exhibit defects in PhANG expression when plants contain well-functioning chloroplasts. Thus, whether these effects of RNAi suppression of HSP90 are caused by specific defects in plastid-to-nucleus signaling or by broad effects on the transcriptome would appear important to test. Indeed, HSP90s broadly contribute to the transcriptome, development, and environmental responses (Sangster et al., 2007). Additionally, the idea that HY5 specifically contributes to Mg-Proto signaling conflicts with the finding that HY5 was a gun mutant when chloroplast biogenesis was blocked with a lincomycin treatment (Ruckle et al., 2007). Lincomycin treatments do not appear to activate the plastid-to-nucleus signaling that depends on tetrapyrroles (Gray et al., 2003; Koussevitzky et al., 2007). Subsequently, phytochrome-associated protein phosphatase 5 (PAPP5)-previously identified as a regulator of phytochrome signaling (Ryu et al., 2005) and as a protein that associates with a Mg-Proto Affi-Gel 102 column (Kindgren et al., 2011)-was demonstrated to serve as a negative regulator of chloroplast function by a variety of assays in green plants not treated with norflurazon. Consistent with this finding Lhcb 2.4 and other PhANGs were expressed at higher levels in papp5 than in wild type. A connection to Mg-Proto signaling was suggested because papp5 specifically increased the levels of chlorophyll in a chlorophyll-deficient Mg-ProtoME cyclase mutant and because the downregulation of Lhcb2.4 expression by exogenous MgProto and exogenous ALA was attenuated in papp5 relative to wild type (Barajas-López et al., 2013). Thus, if Mg-Proto is a plastid signal, potential components of the signaling mechanism are not unique to Mg-Proto signaling. With the addition of ZEITLUPE and PSEUDO-RESPONSE REGULATOR5, this HSP90 and HY5-dependent mechanism was proposed to help plastid signals converge with the circadian clock and regulate the expression of $C$-CBF genes in Arabidopsis (Norén et al., 2016).

Distinct mechanistic information on porphyrin signaling was reported by Zhang et al. (2013). They provided evidence that interactions between porphyrins and GUN1 promote the binding of ABI4 to the promoters of genes that encode Lhcb and CCAAT binding factor A (CBFA). ABI4 was reported to inhibit the transcription of these genes by competitively binding to a ciselement that a distinct transcription activator binds when ABI4 is absent. Additionally, reduced levels of CBFA promoted the accumulation of the LEAFY COTYLEDON1 subunit of the heme activator protein complex (HAP), which binds to promoters of genes encoding HAP and stimulates their transcription. HAP and $\mathrm{CBP}$ induced transcription in general and thus, were proposed to promote the transient increases in the levels of cellular RNA that porphyrin signaling is proposed to induce (Zhang et al., 2013). Although a role for ABI4 acting downstream of GUN1 was repeatedly reported (Koussevitzky et al., 2007; Sun et al., 2011; Zhang et al., 2013; Xu et al., 2016), other work provides evidence that additional nuclear transcription factors contribute to the plastid-to-nucleus signaling that is regulated by the GUN genes, such as HY5 and GLK1 (Acevedo-Hernández et al., 2005; Ruckle et al., 2007; Kakizaki et al., 2009; Waters et al., 2009; Kerchev et al., 2011). 


\section{THE MECHANISM OF TETRAPYRROLE SIGNALING IN PLANTS: HEME}

An alternative to the idea that Mg-Proto serves as plastid signal is that heme serves as a plastid signal. For example, the accumulation and export of heme from the chloroplast to the cytosol or nucleus, or changes in the chloroplastic levels or subchloroplastic location of a particular pool of heme might serve as a plastid signal that induces the expression of PhANGs. In this model, the inductive heme signal is emitted from wellfunctioning chloroplasts and plastids do not emit this inductive signal when chloroplast biogenesis is blocked or attenuated. The main evidence that heme serves as plastid signal is (1) that overexpressing catalytically active $\mathrm{FC} 1$ within the physiologically relevant range and feeding ALA to norflurazon-treated seedlings induced an increase in the expression of PhANGs (Woodson et al., 2011) and (2) that gun5, overexpressing $F C 1$, and exogenous ALA induced the expression of PhANGs in sig2 mutants that were not treated with norflurazon (Woodson et al., 2013). SIG2 is required for the synthesis of tRNA ${ }^{\text {Glu }}$ in plastids (Schön et al., 1986; Kanamaru et al., 2001). The synthesis of glutamyl tRNA from glutamate and tRNA ${ }^{\text {Glu }}$ is the first step in the tetrapyrrole biosynthesis (Tanaka and Tanaka, 2007; Tanaka et al., 2011). The levels tRNA ${ }^{\mathrm{Glu}}$ and glutamyl tRNA were each reduced in sig2, which links SIG2 to tetrapyrrole biosynthesis and signaling (Woodson et al., 2013). Consistent with heme serving as an inductive plastid signal, the expression of ferrochelatase from Bradyrhizobium japonicum in the cytosol of rice was suggested to induce increases in the ALA synthesizing capacity of chloroplasts by activating a signaling mechanism that regulates gene expression in the nucleus (Kim et al., 2014).

There are two isoforms of ferrochelatase in Arabidopsis. The expression of $F C 1$ is observed in non-photosynthetic tissues and is induced by stress. FC2 is coexpressed with PhANGs (Tanaka et al., 2011). Although plants that overexpressed FC1 exhibited gun phenotypes, plants that overexpressed $F C 2$ did not exhibit gun phenotypes (Woodson et al., 2011). These data provide more evidence that plastid-to-nucleus signaling is activated by changes in the location or concentration of a particular pool of a particular porphyrin-perhaps a transient change in concentration-(Mochizuki et al., 2008, 2010; Woodson et al., 2011) and that this porphyrin is heme (Woodson et al., 2011, 2013).

Consistent with heme serving as a plastid signal in plants, chloroplasts are known to synthesize and export heme to a variety of subcellular locations (Thomas and Weinstein, 1990; Cornah et al., 2003; Tanaka et al., 2011), and heme serves as a signal that regulates gene expression in diverse organisms (Tanaka and Hanaoka, 2013; Terry and Smith, 2013; Kobayashi et al., 2016). Nonetheless, there are data that appear to conflict with the idea that porphyrin-dependent plastid-to-nucleus signaling is explained by an increase in the level of heme serving as a plastid signal. For instance, although elevated levels of heme were reported in gun 2 relative to wild type and in gun 5 sig2 relative to sig2, heme was reported to not accumulate above wild-type levels in gun4, gun5, or in plants that overexpress $F C 1$ (Woodson et al.,
2011, 2013; Espinas et al., 2012). There are conflicting data on whether heme accumulates in norflurazon-treated gun2 relative to norflurazon-treated wild type and whether heme accumulates in untreated Arabidopsis seedlings relative to norflurazon-treated seedlings (Voigt et al., 2010; Woodson et al., 2011). The caveat with these conflicting data is that different methods for quantifying the levels of free heme (i.e., heme that is not associated with proteins and that may accumulate in cellular membranes) can yield different results. Indeed, an analysis of methods for extracting and quantifying heme from plants indicated that the level of free heme is not correlated with the gun phenotype. Thus, heme-dependent plastid-to-nucleus signaling does not appear to depend on the accumulation of free heme. The heme that activates plastid-to-nucleus signaling was proposed to bind to a protein that contributes to a signaling mechanism (Espinas et al., 2012).

Although the data favoring a simple heme-based signaling mechanism is compelling, ALA feeding experiments provide evidence that heme signaling is complex or that porphyrin signaling is more complex than a heme signaling mechanism. Although exogenous ALA induced increases in the expression of PhANGs in norflurazon-treated seedlings (Woodson et al., 2011) and although feeding low quantities of ALA to etiolated sig2 and sig6 seedlings induced increases in expression of PhANGs, feeding high quantities of ALA to etiolated sig2 and sig6 seedlings downregulated the expression of $L h c b$. Additionally, feeding low quantities of ALA to etiolated wild-type seedlings had no inductive effect on $L h c b$ expression and feeding larger quantities of ALA to etiolated wild-type seedlings downregulated the expression of Lhcb (Woodson et al., 2013). Exogenous ALA either upregulated or downregulated the expression of PhANG expression depending on the age of Lepidium sativum seedlings grown in far-red light (Kittsteiner et al., 1991). Feeding ALA to both wild-type and $h y 1$ seedlings downregulated the expression of Lhcb in the dark and in far-red light (Vinti et al., 2000; Czarnecki et al., 2012). Feeding hemin to intact plants either downregulated or had no effect on the expression of Lhcb (Zhang et al., 2011a, 2013). Some of the apparently contradictory results from ALA feeding experiments are potentially explained by light-regulated development affecting porphyrin signaling (Woodson et al., 2013). Alternatively, Zhang et al. (2013) suggested that the repressive effect of high concentrations of exogenous ALA and heme on PhANG expression and that the inductive effect of low concentrations of exogenous ALA and the overexpression of $F C 1$ on PhANG expression provides evidence that heme can either downregulate or upregulate the expression of PhANGs depending on whether heme is present at high or low levels in the cell. Although some reports of exogenous ALA downregulating the expression of PhANGs is potentially explained by non-specific and complex effects of unnaturally high levels of porphyrins on PhANG expression, extremely high porphyrin levels would appear necessary to explain a non-specific mechanism or toxicity that is not based on ROS because 200-fold increases in the levels of Mg-Proto and Mg-ProtoME that were induced by exogenous ALA were reported to not affect the expression of $L h c b 1$ (Mochizuki et al., 2008). 
In the conditions used for the gun mutant screen, the effects of the gun 4 and gun 5 alleles on nuclear gene expression are potentially explained by the influence of the defects in Mg-porphyrin biosynthesis on heme biosynthesis (Papenbrock et al., 2000; Alawady and Grimm, 2005; Woodson et al., 2011). Conversely, the data supporting the idea that heme serves as an inductive plastid signal in seedlings containing well-functioning chloroplasts (Woodson et al., 2011, 2013) is also consistent with increased heme biosynthesis affecting the chlorophyll branch of the tetrapyrrole biosynthetic pathway. Perhaps increases in heme biosynthesis might affect the subchloroplastic localization of GUN4, GUN5, or a GUN4-GUN5 complex bound to or not bound to a porphyrin ligand, which might serve as a repressive signal (Woodson et al., 2011).

\section{INTERACTIONS BETWEEN TETRAPYRROLE SIGNALING AND GUN1-DEPENDENT SIGNALING}

The plastid-to-nucleus signaling mechanism that is affected by tetrapyrrole metabolism appears to interact with the plastidto-nucleus signaling that depends on GUN1. Tetrapyrroledependent and GUN1-dependent plastid-to-nucleus signaling mechanisms appeared to downregulate the expression of PhANGs in a partially redundant manner because a synergistic enhancement of the gun phenotype was observed in the gun1-1 gun4-1 and the gun1-1 gun5 double mutants relative to the single mutants (Mochizuki et al., 2001). However, the alleles used by Mochizuki et al. (2001) were leaky. Nonetheless, there was no evidence that the gun phenotype was enhanced in the gun4-1 gun5 double mutant relative to the single mutants (Mochizuki et al., 2001). Subsequently, a double mutant was constructed with gun5 and gun1-9, a non-sense allele of GUN1. The gun phenotype of this double mutant was indistinguishable from gun1-9. These data provide evidence that the tetrapyrrole signal acts upstream of GUN1 (Koussevitzky et al., 2007). The synergistic increase in the gun phenotype observed by Mochizuki et al. (2001) was suggested to result from leaky alleles (Koussevitzky et al., 2007). Transcriptome analyses were also consistent with significant interactions between the plastid signaling mechanisms disrupted by mutant alleles of GUN1 and GUN5 (Koussevitzky et al., 2007). The finding that exogenous Mg-Proto downregulated the expression of $R b c S$ and $L h c b$ in wild type but not in gun1, papp5, abi4, or hy5 (Zhang et al., 2011a; Kindgren et al., 2012; BarajasLópez et al., 2013) provides more evidence that tetrapyrroledependent plastid signaling acts upstream of GUN1. In contrast, exogenous hemin downregulated the expression of a gene that encodes the CBP in gun1-9 in 3-week-old Arabidopsis plants (Zhang et al., 2013). Thus, heme signaling may not absolutely require GUN1 in 3-week-old Arabidopsis plants.

The finding that there were increases in the levels of ALA, heme, and chlorophyll in gun1 sig2 relative to sig2 provides more evidence for interactions between these two plastid-to-nucleus signaling mechanisms (Woodson et al., 2013). Consistent with these and other data, GUN1 was suggested to act upstream of porphyrin biosynthesis and to affect plastid-to-nucleus signaling by serving as a negative regulator of tetrapyrrole biosynthesis (Terry and Smith, 2013). In this model for plastid-to-nucleus signaling, GUN1 acts upstream of tetrapyrrole biosynthesis rather than downstream of tetrapyrrole biosynthesis as previously suggested (Koussevitzky et al., 2007). The finding that although gun1 and gun4-1 suppress the chlorophyll-deficient phenotype of slow green1, gun5 enhances the chlorophyll-deficient phenotype of slow green1 (Hu et al., 2014) provides evidence for a complex interaction between tetrapyrrole metabolism and GUN1. The finding that light-regulated development affects porphyrindependent plastid-to-nucleus signaling (Woodson et al., 2013) indicates additional complexity in this type of plastid-to-nucleus signaling.

In general, pentatricopeptide repeat proteins, such as GUN1, contribute to the expression of the plastidic and mitochondrial genomes (Barkan and Small, 2014). The simplest interpretation of the current data is that the influence of GUN1 on plastid-to-nucleus signaling is probably related to a role for GUN1 in the expression of the chloroplast genome and that GUN1 and tetrapyrrole metabolism interact by an indirect mechanism because the expression of the chloroplast genome and tetrapyrrole metabolism are distinct processes. However, direct interactions between GUN1 and tetrapyrrole metabolism seem possible because the biochemical function of GUN1 is poorly understood and because there is considerable genetic evidence for interactions between GUN1 and tetrapyrrole metabolism. Heme was suggested to possibly interact with GUN1-dependent plastid-to-nucleus signaling by affecting PHD type transcription factor with transmembrane domains (PTM), which acts downstream of GUN1 (Sun et al., 2011; Terry and Smith, 2013).

One approach for learning more about the biochemical function of GUN1 and the interactions between GUN1 and tetrapyrrole metabolism is to identify other molecules that bind GUN1, such as other proteins. However, purifying a multisubunit protein complex containing native GUN1 is potentially difficult because GUN1 is thought to accumulate at low levels (Liu et al., 2013). Tadini et al. (2016) provided evidence that GUN1 and the enzymes that perform tetrapyrrole biosynthesis might directly interact by performing two hybrid and BiFC experiments and by purifying a GFP-tagged version of GUN1 from plants that overexpressed GUN1-GFP after treating chloroplasts that were purified from these plants with a crosslinking agent. GUN1 is capable of interacting with four proteins that contribute to tetrapyrrole biosynthesis-the $79-\mathrm{kDa}$ subunit of Mg-chelatase (also known as ChlD), porphobilinogen deaminase, uroporphyrinogen III decarboxylase, and ferrochelatase I-when these proteins are expressed at abnormally high levels in twohybrid and BiFC experiments. Nearly 300 different proteins that contribute to diverse chloroplastic processes were crosslinked to GUN1-GFP after GUN1-GFP was overexpressed in Arabidopsis. One of these crosslinked proteins was ChlD. Thus, GUN1 has a propensity for interacting with many other proteins when it is overexpressed in yeast and plants (Tadini et al., 2016). The relevance of these numerous interactions for native GUN1 expressed at normal levels in wild-type plants is not known. 


\section{BIOLOGICAL FUNCTION OF TETRAPYRROLE SIGNALING IN PLANTS}

The finding that in addition to Arabidopsis, both maize and barley mutants that are deficient in Mg-chelatase exhibited gun phenotypes (Burgess and Taylor, 1988; Gadjieva et al., 2005) indicates that selection pressure has maintained this signaling mechanism in diverse plants and that the influence of tetrapyrrole metabolism on plastid-to-nucleus signaling serves an important function in plants. Nonetheless, the biological function of tetrapyrrole-dependent plastid-to-nucleus signaling is not well established.

Although the influence of tetrapyrrole-dependent plastid-tonucleus signaling on the expression of PhANGs is commonly studied, transcriptome analyses of $C$. reinhardtii are consistent with tetrapyrrole-dependent plastid-to-nucleus signaling not serving as a major regulator of PhANG expression. Indeed, the largest group of genes regulated by $\mathrm{Mg}$-Proto and hemin in C. reinhardtii contribute to proteolysis and protein folding. The expression of more than half of these genes is regulated by heat shock. Based on these data, porphyrin signaling was proposed to help $C$. reinhardtii adapt to changing environmental conditions (Voß et al., 2011). The finding that the overexpression of stress-inducible $F C 1$ but not the overexpression of photosynthesis-associated $F C 2$ affects plastid-to-nucleus signaling in Arabidopsis is consistent with porphyrin-dependent plastid-to-nucleus signaling affecting nonphotosynthetic and stress-related functions in Arabidopsis (Woodson et al., 2011). Nonetheless, transcriptome analyses provided evidence that porphyrin-dependent plastid-to-nucleus signaling affects PhANG expression in Arabidopsis (Strand et al., 2003). Unfortunately, a plant system similar to C. reinhardtii in the sense that tetrapyrrole-dependent plastid-to-nucleus signaling is rapidly induced without introducing complicating secondary effects, such as ROS, does not exist.

Tetrapyrrole-dependent plastid-to-nucleus signaling may broadly affect plant cells. Heme signaling appears to affect the expression of genes that contribute to starch biosynthesis and the accumulation of starch during amyloplast biogenesis in BY2 cells (Enami et al., 2011). gun5 has less basal thermotolerance than wild type (Miller et al., 2007). Porphyrin signaling in general was suggested to promote oxidative stress tolerance (Zhang et al., 2011b). gun5 and cch exhibit reduced cold tolerance relative to wild type (Kindgren et al., 2015). Overexpressing Myxococcus xanthus protoporphyrinogen IX oxidase in Oryza sativa enhanced drought tolerance relative to wild type (Phung et al., 2011). Although Phung et al. (2011) proposed that overexpressing protoporphyrinogen IX oxidase promoted drought tolerance by inducing increases in the levels of ROS that helped acclimate chloroplasts to stress, they did not detect elevated levels of ROS in plants that overexpressed protoporphyrinogen IX oxidase. Additionally, the lines overexpressing protoporphyrinogen IX oxidase exhibited an increased capacity for porphyrin biosynthesis that resulted in elevated levels of heme. Interesting, their data show a correlation between elevated levels of ferrochelatase activity, heme, and Lhcb expression, although the levels of heme appeared to increase only transiently (Phung et al., 2011). The data from Phung et al. (2011) are consistent with tetrapyrrole signaling, such as heme signaling, promoting drought tolerance. Indeed, there is a growing body of evidence supporting a role for heme signaling in drought tolerance in plants (Nagahatenna et al., 2015). Porphyrin-derived ROS, heme signaling, or both may contribute to drought tolerance in the transgenic plants described by Phung et al. (2011). A mutant allele that specifically disrupts tetrapyrrole-dependent plastid signaling without directly affecting an enzyme that contributes to tetrapyrrole biosynthesis would help to establish the biological function of tetrapyrrole-dependent plastid-to-nucleus signaling.

The interactions between GUN1- and tetrapyrrole-dependent plastid-to-nucleus signaling are consistent with additional biological functions for tetrapyrrole-dependent plastid-tonucleus signaling because GUN1 is known to promote diverse biological functions in plants. Loss-of-function alleles of GUN1 affect chloroplast biogenesis (Mochizuki et al., 1996; Ruckle et al., 2007), plastid-to-nucleus signaling when plants are grown in diverse conditions (Susek et al., 1993; Koussevitzky et al., 2007; Ruckle et al., 2007; Kakizaki et al., 2009; Woodson et al., 2013; Tanaka et al., 2015; Tadini et al., 2016), the circadian rhythm (Hassidim et al., 2007), the accumulation of anthocyanins (Ruckle and Larkin, 2009; Cottage et al., 2010; Cheng et al., 2012), abiotic stress tolerance (Miller et al., 2007; Zhang et al., 2013), and the development of seedlings and leaves (Ruckle and Larkin, 2009; Cottage et al., 2010; Tameshige et al., 2013; Martín et al., 2016; Xu et al., 2016).

\section{SUMMARY}

Since the early days of plastid-to-nucleus signaling research, porphyrins were attractive candidates for plastid signals that regulate nuclear gene expression. A number of independent experiments with exogenous ALA, exogenous porphyrins, inhibitors, a variety of mutants with defects in tetrapyrrole metabolism, and both the overexpression and antisense suppression of enzymes that contribute to tetrapyrrole biosynthesis provide a large body of evidence favoring the idea that porphyrins regulate the expression of nuclear genes. Results from the gun mutant screen indicate that when chloroplast biogenesis is blocked, tetrapyrrole metabolism regulates the expression of PhANGs. There is a large body of evidence indicating that in the conditions used for the gun mutant screen, the effects of tetrapyrrole metabolism on nuclear gene expression are difficult or impossible to explain by changes in the levels of porphyrin-derived ROS. The regulation of nuclear gene expression by tetrapyrrole metabolism is conserved in Arabidopsis, barely, and maize. Thus, tetrapyrrole signaling makes an important contribution to plants.

The simplest interpretation of the current data is that in the conditions used for the gun mutant screen, heme serves as a plastid signal that regulates nuclear gene expression. The current data are also consistent with other mechanisms, such as a moonlighting function (Boukouris et al., 2016) 


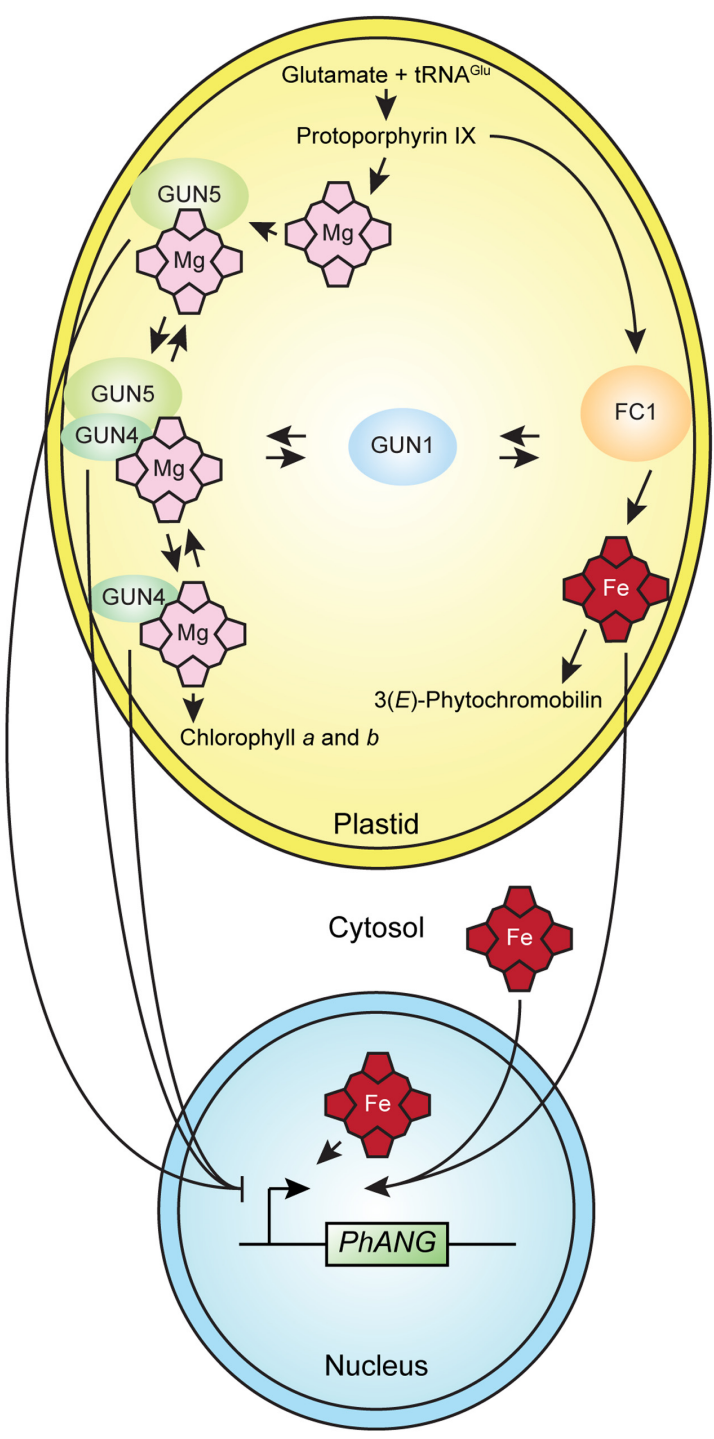

FIGURE 3 | A model for tetrapyrrole-dependent plastid-to-nucleus signaling in plants. The simplest interpretation of the current data is that heme accumulates in well-functioning chloroplasts and that the accumulation of heme in chloroplasts or the export of heme to the cytosol or nucleus activates a mechanism that induces the expression of PhANGs. The current data do not exclude alternative mechanisms, such as a moonlighting function for an enzyme or regulatory factor that contributes to tetrapyrrole metabolism, such as GUN4, GUN5, or a GUN4-GUN5 complex that affects gene expression in the nucleus. Either the liganded or unliganded versions of GUN4, GUN5, or a GUN4-GUN5 complex might contribute to a plastid-to-nucleus signaling mechanism that regulates the expression of PhANGs. GUN1 interacts with tetrapyrrole-dependent plastid-to-nucleus signaling by an unknown mechanism.

for an enzyme or regulatory factor that contributes to tetrapyrrole metabolism in plastid-to-nucleus signaling, such as GUN5 (Mochizuki et al., 2001), GUN4, or a GUN4-GUN5 complex (Larkin et al., 2003) in plastid-to-nucleus signaling (Figure 3). The current data are also consistent with changes in the flux through the tetrapyrrole biosynthetic pathway activating a plastid-to-nucleus signaling mechanism (Mochizuki et al., 2008). There are precedents for each of these mechanisms in non-plant systems (Osanai et al., 2009; Terry and Smith, 2013). Interactions between tetrapyrroledependent plastid-to-nucleus signaling and GUN1-dependent plastid-to-nucleus signaling potentially link tetrapyrroledependent plastid-to-nucleus signaling to a network of plastidic and extraplastidic signaling mechanisms that is broadly significant.

The plastid-to-nucleus signaling mechanism that is specifically interrogated by the gun mutant screen in young seedlings does not appear to require a transient or sustained accumulation of Mg-Proto or Mg-ProtoME. However, in mature plants, the photooxidative stress that (1) induces increases in the levels of Mg-Proto and Mg-ProtoME and (2) is correlated with downregulated expression of PhANGs was observed under distinct developmental and environmental conditions relative to the conditions used to screen for gun mutants. The evidence consistent with oxidative stress inducing an increase in the levels of $\mathrm{Mg}$-Proto or $\mathrm{Mg}$-ProtoME that affects gene expression in the nucleus is also consistent with oxidative stress attenuating heme signaling or at least in some instances, ROS signaling

At this point, it is perhaps most important to understand the mechanism of tetrapyrrole signaling in plants. Understanding this mechanism may help us to understand why perturbations in tetrapyrrole metabolism sometimes upregulate, downregulate, or have no effect on the expression of nuclear genes. Understanding this mechanism may help us to understand the interactions between tetrapyrrole signaling and GUN1-dependent plastidto-nucleus signaling, interactions between tetrapyrrole signaling and light-regulated development, and the biological function of tetrapyrrole signaling. Identifying a protein that specifically contributes to tetrapyrrole signaling (e.g., a protein that serves as a heme receptor in the plastid, cytosol, or nucleus) is perhaps the highest priority. Mutants with defects in such a gene are essential for learning the biological function of tetrapyrrole signaling in plants. The evidence for tetrapyrrole signaling contributing to abiotic stress tolerance and for the interactions between tetrapyrrole metabolism and GUN1-dependent plastidto-to-nucleus signaling are consistent with an important role for tetrapyrrole signaling in plants. Thus, future research in this area is expected to advance our understanding of plant biology and possibly contribute to the development of new germplasm that will help to meet the needs of agriculture in our changing environment.

\section{AUTHOR CONTRIBUTIONS}

The author confirms being the sole contributor of this work and approved it for publication.

\section{ACKNOWLEDGMENTS}

Research in the author's laboratory is supported by Huazhong Agricultural University, Wuhan, China. 


\section{REFERENCES}

Aarti, D. P., Tanaka, R., and Tanaka, A. (2006). Effects of oxidative stress on chlorophyll biosynthesis in cucumber (Cucumis sativus) cotyledons. Physiol. Plant. 128, 186-197. doi: 10.1111/j.1399-3054.2006.00720.x

Acevedo-Hernández, G. J., León, P., and Herrera-Estrella, L. R. (2005). Sugar and ABA responsiveness of a minimal RBCS light-responsive unit is mediated by direct binding of ABI4. Plant J. 43, 506-519. doi: 10.1111/j.1365313X.2005.02468.X

Adhikari, N. D., Froehlich, J. E., Strand, D. D., Buck, S. M., Kramer, D. M., and Larkin, R. M. (2011). GUN4-porphyrin complexes bind the ChlH/GUN5 subunit of Mg-chelatase and promote chlorophyll biosynthesis in Arabidopsis. Plant Cell 23, 1449-1467. doi: 10.1105/tpc.110.082503

Adhikari, N. D., Orler, R., Chory, J., Froehlich, J. E., and Larkin, R. M. (2009). Porphyrins stabilize the association of GENOMES UNCOUPLED 4 and a Mgchelatase subunit with chloroplast membranes. J. Biol. Chem. 284, 24783-24796. doi: 10.1074/jbc.M109.025205

Alawady, A. E., and Grimm, B. (2005). Tobacco Mg protoporphyrin IX methyltransferase is involved in inverse activation of $\mathrm{Mg}$ porphyrin and protoheme synthesis. Plant J. 41, 282-290. doi: 10.1111/j.1365-313X.2004.02291.x

Ankele, E., Kindgren, P., Pesquet, E., and Strand, Å. (2007). In vivo visualization of Mg-protoporphyrin IX, a coordinator of photosynthetic gene expression in the nucleus and the chloroplast. Plant Cell 19, 1964-1979. doi: $10.1105 /$ tpc. 106.048744

Armbruster, U., Pesaresi, P., Pribil, M., Hertle, A., and Leister, D. (2011). Update on chloroplast research: new tools, new topics, and new trends. Mol. Plant 4, 1-16. doi: $10.1093 / \mathrm{mp} / \mathrm{ssq} 060$

Barajas-López, J., Kremnev, D., Shaikhali, J., Piñas-Fernández, A., and Strand, A. (2013). PAPP5 is involved in the tetrapyrrole mediated plastid signalling during chloroplast development. PLoS ONE 8:e60305. doi: 10.1371/journal.pone.0060305

Barajas-López, J. D. D., Blanco, N. E., and Strand, Å. (2012). Plastid-to-nucleus communication, signals controlling the running of the plant cell. Biochim. Biophys. Acta 1833, 425-437. doi: 10.1016/j.bbamcr.2012.06.020

Barkan, A., and Small, I. (2014). Pentatricopeptide repeat proteins in plants. Annu. Rev. Plant Biol. 65, 415-442. doi: 10.1146/annurev-arplant-050213-040159

Beck, C. F. (2005). Signaling pathways from the chloroplast to the nucleus. Planta 222, 743-756. doi: 10.1007/s00425-005-0021-2

Bobik, K., and Burch-Smith, T. M. (2015). Chloroplast signaling within, between and beyond cells. Front. Plant Sci. 6:781. doi: 10.3389/fpls.2015.00781

Boukouris, A. E., Zervopoulos, S. D., and Michelakis, E. D. (2016). Metabolic enzymes moonlighting in the nucleus: metabolic regulation of gene transcription. Trends Biochem. Sci. doi: 10.1016/j.tibs.2016.05.013 [Epub ahead of print].

Bradbeer, J. W., Atkinson, Y. E., Börner, T., and Hagemann, R. (1979). Cytoplasmic synthesis of plastid polypeptides may be controlled by plastid-synthesised RNA. Nature 279, 816-817. doi: 10.1038/279816a0

Brown, N. J., Sullivan, J. A., and Gray, J. C. (2005). Light and plastid signals regulate the expression of the pea plastocyanin gene through a common region at the $5^{\prime}$ end of the coding region. Plant J. 43, 541-552. doi: 10.1111/j.1365313X.2005.02474.X

Brzezowski, P., Richter, A. S., and Grimm, B. (2015). Regulation and function of tetrapyrrole biosynthesis in plants and algae. Biochim. Biophys. Acta 1847, 968-985. doi: 10.1016/j.bbabio.2015.05.007

Brzezowski, P., Schlicke, H., Richter, A., Dent, R. M., Niyogi, K. K., and Grimm, B. (2014). The GUN4 protein plays a regulatory role in tetrapyrrole biosynthesis and chloroplast-to-nucleus signalling in Chlamydomonas reinhardtii. Plant J. 79, 285-298. doi: 10.1111/tpj.12560

Burgess, D. G., and Taylor, W. C. (1988). The chloroplast affects the transcription of a nuclear gene family. Mol. Gen. Genet. 214, 89-96. doi: 10.1007/BF00340185

Chan, K. X., Phua, S. Y., Crisp, P., McQuinn, R., and Pogson, B. J. (2016). Learning the languages of the chloroplast: retrograde signaling and beyond. Annu. Rev. Plant Biol. 67, 25-53. doi: 10.1146/annurev-arplant-043015-111854

Chen, X., Pu, H., Wang, X., Long, W., Lin, R., and Liu, L. (2015). Crystal structures of GUN4 in complex with porphyrins. Mol. Plant 8, 1125-1127. doi: 10.1016/j.molp.2015.04.013
Cheng, J., Yuan, S., Zhang, Z. W., Zhu, F., Tang, H., Xu, F., et al. (2012). Plastidsignalling-mediated anthocyanin accumulation in mature Arabidopsis rosettes. Plant Growth Regul. 68, 223-230. doi: 10.1007/s10725-012-9710-6

Cornah, J. E., Terry, M. J., and Smith, A. G. (2003). Green or red: what stops the traffic in the tetrapyrrole pathway? Trends Plant Sci. 8, 224-230. doi: 10.1016/S1360-1385(03)00064-5

Cottage, A., Mott, E. K., Kempster, J. A., and Gray, J. C. (2010). The Arabidopsis plastid-signalling mutant gun 1 (genomes uncoupled1) shows altered sensitivity to sucrose and abscisic acid and alterations in early seedling development. J. Exp. Bot. 61, 3773-3786. doi: 10.1093/jxb/erq186

Cottage, A. J., Mott, E. K., Wang, J.-H., Sullivan, J. A., MacLean, D., Tran, L., et al. (2008). "GUN1 (GENOMES UNCOUPLED1) encodes a pentatricopeptide repeat (PPR) protein involved in plastid protein synthesisresponsive retrograde signaling to the nucleus," in Photosynthesis. Energy from the Sun: 14th International Congress on Photosynthesis, eds J. F. Allen, E. Gnatt, J. H. Golbeck, and B. Osmond (Berlin: Springer), 1201-1205.

Czarnecki, O., Gläßer, C., Chen, J. G., Mayer, K. F., and Grimm, B. (2012). Evidence for a contribution of ALA synthesis to plastid-to-nucleus signaling. Front. Plant Sci. 3:236. doi: 10.3389/fpls.2012.00236

Davison, P. A., Schubert, H. L., Reid, J. D., Iorg, C. D., Heroux, A., Hill, C. P., et al. (2005). Structural and biochemical characterization of Gun4 suggests a mechanism for its role in chlorophyll biosynthesis. Biochemistry 44, 7603-7612. doi: 10.1021/bi050240x

Enami, K., Ozawa, T., Motohashi, N., Nakamura, M., Tanaka, K., and Hanaoka, M. (2011). Plastid-to-nucleus retrograde signals are essential for expression of nuclear starch biosynthesis genes during amyloplast differentiation in tobacco BY-2 cultured cells. Plant Physiol. 157, 518-530. doi: 10.1104/pp.111.178897

Espinas, N. A., Kobayashi, K., Takahashi, S., Mochizuki, N., and Masuda, T. (2012). Evaluation of unbound free heme in plant cells by differential acetone extraction. Plant Cell Physiol. 53, 1344-1354. doi: 10.1093/pcp/pcs067

Formighieri, C., Ceol, M., Bonente, G., Rochaix, J. D., and Bassi, R. (2012). Retrograde signaling and photoprotection in a gun 4 mutant of Chlamydomonas reinhardtii. Mol. Plant 5, 1242-1262. doi: 10.1093/mp/sss051

Forsburg, S. L., and Guarente, L. (1989). Communication between mitochondria and the nucleus in regulation of cytochrome genes in the yeast Saccharomyces cerevisiae. Annu. Rev. Cell Biol. 5, 153-180. doi: 10.1146/annurev.cb.05.110189.001101

Gadjieva, R., Axelsson, E., Olsson, U., and Hansson, M. (2005). Analysis of gun phenotype in barley magnesium chelatase and Mg-protoporphyrin IX monomethyl ester cyclase mutants. Plant Physiol. Biochem. 43, 901-908. doi: 10.1016/j.plaphy.2005.08.003

Galvez-Valdivieso, G., and Mullineaux, P. M. (2010). The role of reactive oxygen species in signalling from chloroplasts to the nucleus. Physiol. Plant. 138, 430-439. doi: 10.1111/j.1399-3054.2009.01331.x

Gough, S. (1972). Defective synthesis of porphyrins in barley plastids caused by mutation in nuclear genes. Biochim. Biophys. Acta 286, 36-54. doi: 10.1016/0304-4165(72)90086-4

Granick, S. (1959). Magnesium porphyrins formed by barley seedlings treated with d-aminolevulinic acid. Plant Physiol. 34:XVIII.

Gray, J. C., Sullivan, J. A., Wang, J. H., Jerome, C. A., and MacLean, D. (2003). Coordination of plastid and nuclear gene expression. Philos. Trans. R. Soc. Lond. B Biol. Sci. 358, 135-144. doi: 10.1098/rstb.2002.1180

Hassidim, M., Yakir, E., Fradkin, D., Hilman, D., Kron, I., Keren, N., et al. (2007). Mutations in CHLOROPLAST RNA BINDING provide evidence for the involvement of the chloroplast in the regulation of the circadian clock in Arabidopsis. Plant J. 51, 551-562. doi: 10.1111/j.1365-313X.2007.03160.x

Hu, Z., Xu, F., Guan, L., Qian, P., Liu, Y., Zhang, H., et al. (2014). The tetratricopeptide repeat-containing protein slow green1 is required for chloroplast development in Arabidopsis. J. Exp. Bot. 65, 1111-1123. doi: $10.1093 / \mathrm{jxb} / \mathrm{ert} 463$

Huang, Y. S., and Li, H. M. (2009). Arabidopsis CHLI2 can substitute for CHLI1. Plant Physiol. 150, 636-645. doi: 10.1104/pp.109.135368

Jansson, S., Pichersky, E., Bassi, R., Green, B. R., Ikeuchi, M., Melis, A., et al. (1992). A nomenclature for the genes encoding the chlorophyll a/b-binding proteins of higher plants. Plant Mol. Biol. Rep. 10, 242-253. doi: 10.1007/BF02668357

Jarvis, P., and López-Juez, E. (2013). Biogenesis and homeostasis of chloroplasts and other plastids. Nat. Rev. Mol. Cell Biol. 14, 787-802. doi: 10.1038/nrm3702 
Johanningmeier, U., and Howell, S. H. (1984). Regulation of light-harvesting chlorophyll-binding protein mRNA accumulation in Chlamydomonas reinhardtii. Possible involvement of chlorophyll synthesis precursors. J. Biol. Chem. 259, 13541-13549.

Kakizaki, T., Matsumura, H., Nakayama, K., Che, F. S., Terauchi, R., and Inaba, T. (2009). Coordination of plastid protein import and nuclear gene expression by plastid-to-nucleus retrograde signaling. Plant Physiol. 151, 1339-1353. doi: 10.1104/pp.109.145987

Kanamaru, K., Nagashima, A., Fujiwara, M., Shimada, H., Shirano, Y., Nakabayashi, K., et al. (2001). An Arabidopsis sigma factor (SIG2)-dependent expression of plastid-encoded tRNAs in chloroplasts. Plant Cell Physiol. 42, 1034-1043. doi: $10.1093 /$ pcp/pce155

Kerchev, P. I., Pellny, T. K., Vivancos, P. D., Kiddle, G., Hedden, P., Driscoll, S., et al. (2011). The transcription factor ABI4 is required for the ascorbic acid-dependent regulation of growth and regulation of jasmonate-dependent defense signaling pathways in Arabidopsis. Plant Cell 23, 3319-3334. doi: 10.1105/tpc.111.090100

Kim, C., and Apel, K. (2013). 1O2-mediated and EXECUTER dependent retrograde plastid-to-nucleus signaling in norflurazon-treated seedlings of Arabidopsis thaliana. Mol. Plant 6, 1580-1591. doi: $10.1093 / \mathrm{mp} / \mathrm{sst} 020$

Kim, J. G., Back, K., Lee, H. Y., Lee, H. J., Phung, T. H., Grimm, B., et al. (2014). Increased expression of Fe-chelatase leads to increased metabolic flux into heme and confers protection against photodynamically induced oxidative stress. Plant Mol. Biol. 86, 271-287. doi: 10.1007/s11103-014-0228-3

Kindgren, P., Dubreuil, C., and Strand, Å. (2015). The recovery of plastid function is required for optimal response to low temperatures in Arabidopsis. PLoS ONE 10:e0138010. doi: 10.1371/journal.pone.0138010

Kindgren, P., Eriksson, M., Benedict, C., Mohapatra, A., Gough, S. P., Hansson, M., et al. (2011). A novel proteomic approach reveals a role for $\mathrm{Mg}$-protoporphyrin IX in response to oxidative stress. Physiol. Plant. 141, 310-320. doi: 10.1111/j.1399-3054.2010.01440.x

Kindgren, P., Norén, L., López, J. D., Shaikhali, J., and Strand, A (2012). Interplay between Heat Shock Protein 90 and HY 5 controls PhANG expression in response to the GUN5 plastid signal. Mol. Plant 5, 901-913. doi: $10.1093 / \mathrm{mp} / \mathrm{ssr} 112$

Kittsteiner, U., Brunner, H., and Rüdiger, W. (1991). The greening process in cress seedlings. II. Complexing agents and 5-aminolevulinate inhibit accumulation of cab-mRNA coding for the light-harvesting chlorophyll a/b protein. Physiol. Plant. 81, 190-196. doi: 10.1111/j.1399-3054.1991.tb01726.x

Kleine, T., and Leister, D. (2013). Retrograde signals galore. Front. Plant Sci. 4:45. doi: $10.3389 /$ fpls.2013.00045

Kleine, T., Voigt, C., and Leister, D. (2009). Plastid signalling to the nucleus: messengers still lost in the mists? Trends Genet. 25, 185-192. doi: 10.1016/j.tig.2009.02.004

Kobayashi, K., Mochizuki, N., Yoshimura, N., Motohashi, K., Hisabori, T., and Masuda, T. (2008). Functional analysis of Arabidopsis thaliana isoforms of the Mg-chelatase CHLI subunit. Photochem. Photobiol. Sci. 7, 1188-1195. doi: $10.1039 / \mathrm{b} 802604 \mathrm{c}$

Kobayashi, Y., Hiroyuki, A., Hanaoka, M., and Tanaka, K. (2016). Abscisic acid participates in the control of cell cycle initiation through heme homeostasis in the unicellular red alga Cyanidioschyzon merolae. Plant Cell Physiol. 57, 953-960. doi: 10.1093/pcp/pcw054

Kobayashi, Y., Kanesaki, Y., Tanaka, A., Kuroiwa, H., Kuroiwa, T., and Tanaka, K. (2009). Tetrapyrrole signal as a cell-cycle coordinator from organelle to nuclear DNA replication in plant cells. Proc. Natl. Acad. Sci. U.S.A. 106, 803-807. doi: 10.1073/pnas.0804270105

Koncz, C., Mayerhofer, R., Koncz-Kalman, Z., Nawrath, C., Reiss, B., Redei, G. P., et al. (1990). Isolation of a gene encoding a novel chloroplast protein by T-DNA tagging in Arabidopsis thaliana. EMBO J. 9, 1337-1346.

Koornneef, M., Rolff, E., and Spruit, C. J. P. (1980). Genetic control of lightinhibited hypocotyl elongation in Arabidopsis thaliana (L) Heynh. Z. Pflanzenphysiol. 100, 147-160. doi: 10.1016/S0044-328X(80)80208-X

Koussevitzky, S., Nott, A., Mockler, T. C., Hong, F., Sachetto-Martins, G., Surpin, M., et al. (2007). Signals from chloroplasts converge to regulate nuclear gene expression. Science 316, 715-719. doi: 10.1126/science.\%201140516

Kropat, J., Oster, U., Rüdiger, W., and Beck, C. F. (2000). Chloroplast signalling in the light induction of nuclear HSP70 genes requires the accumulation of chlorophyll precursors and their accessibility to cytoplasm/nucleus. Plant J. 24, 523-531. doi: 10.1046/j.1365-313x.2000.00898.x

La Rocca, N., Rascio, N., Oster, U., and Rüdiger, W. (2001). Amitrole treatment of etiolated barley seedlings leads to deregulation of tetrapyrrole synthesis and to reduced expression of Lhc and RbcS genes. Planta 213, 101-108. doi: $10.1007 / \mathrm{s} 004250000477$

Larkin, R. M. (2014). Influence of plastid signals on light signaling and development. Philos. Trans. R. Soc. Lond. B Biol. Sci. 369, 20130232. doi: 10.1098/rstb.2013.0232

Larkin, R. M., Alonso, J. M., Ecker, J. R., and Chory, J. (2003). GUN4, a regulator of chlorophyll synthesis and intracellular signaling. Science 299, 902-906. doi: 10.1126/science. 1079978

Larkin, R. M., Stefano, G., Ruckle, M. E., Stavoe, A. K. H., Sinkler, C., Brandizzi, F., et al. (2016). REDUCED CHLOROPLAST COVERAGE genes from Arabidopsis thaliana help to establish the size of the chloroplast compartment. Proc. Natl. Acad. Sci. U.S.A. 113, E1116-E1125. doi: 10.1073/pnas.1515741113

Lepistö, A., and Rintamäki, E. (2012). Coordination of plastid and light signaling pathways upon development of Arabidopsis leaves under various photoperiods. Mol. Plant 5, 799-816. doi: 10.1093/mp/ssr106

Liu, S., Melonek, J., Boykin, L. M., Small, I., and Howell, K. A. (2013). PPRSMRs: ancient proteins with enigmatic functions. RNA Biol. 10, 1501-1510. doi: 10.4161/rna.26172

Martín, G., Leivar, P., Ludevid, D., Tepperman, J. M., Quail, P. H., and Monte, E. (2016). Phytochrome and retrograde signalling pathways converge to antagonistically regulate a light-induced transcriptional network. Nat. Commun. 7, 11431. doi: 10.1038/ncomms11431

Mascia, P. (1978). An analysis of precursors accumulated by several chlorophyll biosynthetic mutants of maize. Mol. Gen. Genet. 167, 237-244. doi: 10.1007/BF00330996

Meinecke, L., Alawady, A., Schroda, M., Willows, R., Kobayashi, M. C., Niyogi, K. K., et al. (2010). Chlorophyll-deficient mutants of Chlamydomonas reinhardtii that accumulate magnesium protoporphyrin IX. Plant Mol. Biol. 72, 643-658. doi: 10.1007/s11103-010-9604-9

Miller, G., Suzuki, N., Rizhsky, L., Hegie, A., Koussevitzky, S., and Mittler, R. (2007). Double mutants deficient in cytosolic and thylakoid ascorbate peroxidase reveal a complex mode of interaction between reactive oxygen species, plant development, and response to abiotic stresses. Plant Physiol. 144, 1777-1785. doi: 10.1104/pp.107.101436

Mochizuki, N., Brusslan, J. A., Larkin, R., Nagatani, A., and Chory, J. (2001). Arabidopsis genomes uncoupled 5 (GUN5) mutant reveals the involvement of Mg-chelatase $\mathrm{H}$ subunit in plastid-to-nucleus signal transduction. Proc. Natl. Acad. Sci. U.S.A. 98, 2053-2058. doi: 10.1073/pnas.98.4.2053

Mochizuki, N., Susek, R., and Chory, J. (1996). An intracellular signal transduction pathway between the chloroplast and nucleus is involved in de-etiolation. Plant Physiol. 112, 1465-1469. doi: 10.1104/pp.112.4.1465

Mochizuki, N., Tanaka, R., Grimm, B., Masuda, T., Moulin, M., Smith, A. G., et al. (2010). The cell biology of tetrapyrroles: a life and death struggle. Trends Plant Sci. 15, 488-498. doi: 10.1016/j.tplants.2010.05.012

Mochizuki, N., Tanaka, R., Tanaka, A., Masuda, T., and Nagatani, A. (2008). The steady-state level of Mg-protoporphyrin IX is not a determinant of plastid-tonucleus signaling in Arabidopsis. Proc. Natl. Acad. Sci. U.S.A. 105, 15184-15189. doi: 10.1073/pnas.0803245105

Moulin, M., McCormac, A. C., Terry, M. J., and Smith, A. G. (2008). Tetrapyrrole profiling in Arabidopsis seedlings reveals that retrograde plastid nuclear signaling is not due to Mg-protoporphyrin IX accumulation. Proc. Natl. Acad. Sci. U.S.A. 105, 15178-15183. doi: 10.1073/pnas.0803054105

Nagahatenna, D. S., Langridge, P., and Whitford, R. (2015). Tetrapyrrolebased drought stress signalling. Plant Biotechnol. J. 13, 447-459. doi: $10.1111 /$ pbi. 12356

Nisar, N., Li, L., Lu, S., Khin, N. C., and Pogson, B. J. (2015). Carotenoid metabolism in plants. Mol. Plant 8, 68-82. doi: 10.1016/j.molp.2014.12.007

Norén, L., Kindgren, P., Stachula, P., Rühl, M., Eriksson, M. E., Hurry, V., et al. (2016). Circadian and plastid signaling pathways are integrated to ensure correct expression of the CBF and COR genes during photoperiodic growth. Plant Physiol. 171, 1392-1406. doi: 10.1104/pp.16.00374

Osanai, T., Imashimizu, M., Seki, A., Sato, S., Tabata, S., Imamura, S., et al. (2009). $\mathrm{ChlH}$, the $\mathrm{H}$ subunit of the $\mathrm{Mg}$-chelatase, is an anti-sigma factor for SigE in 
Synechocystis sp. PCC 6803. Proc. Natl. Acad. Sci. U.S.A. 106, 6860-6865. doi: 10.1073/pnas. 0810040106

Oster, U., Brunner, H., and Rüdiger, W. (1996). The greening process in cress seedlings. V. Possible interference of chlorophyll precursors, accumulated after thujaplicin treatment, with light-regulated expression of Lhc genes. J. Photochem. Photobiol. B 36, 255-261. doi: 10.1016/S1011-1344(96)07388-5

Osteryoung, K. W., and Pyke, K. A. (2014). Plastid and proplastid division. Ann. Rev. Plant. Biol. 65, 443-472. doi: 10.1146/annurev-arplant-050213-035748

Papenbrock, J., Mock, H.-P., Kruse, E., and Grimm, B. (1999). Expression studies in tetrapyrrole biosynthesis: inverse maxima of magnesium chelatase and ferrochelatase activity during cyclic photoperiods. Planta 208, 264-273. doi: $10.1007 / \mathrm{s} 004250050558$

Papenbrock, J., Pfündel, E., Mock, H. P., and Grimm, B. (2000). Decreased and increased expression of the subunit CHL I diminishes Mg chelatase activity and reduces chlorophyll synthesis in transgenic tobacco plants. Plant J. 22, 155-164. doi: 10.1046/j.1365-313x.2000.00724.x

Paulsen, H., and Bogorad, L. (1988). Diurnal and circadian rhythms in the accumulation and synthesis of mRNA for the light-harvesting Chlorophyll a/b-Binding protein in tobacco. Plant Physiol. 88, 1104-1109. doi: 10.1104/pp.88.4.1104

Pfannschmidt, T. (2010). Plastidial retrograde signalling - a true "plastid factor" or just metabolite signatures? Trends Plant Sci. 15, 427-435. doi: 10.1016/.j.tplants.2010.05.009

Phung, T. H., Jung, H. I., Park, J. H., Kim, J. G., Back, K., and Jung, S. (2011). Porphyrin biosynthesis control under water stress: sustained porphyrin status correlates with drought tolerance in transgenic rice. Plant Physiol. 157, 17461764. doi: $10.1104 /$ pp. 111.188276

Pogson, B. J., Ganguly, D., and Albrecht-Borth, V. (2015). Insights into chloroplast biogenesis and development. Biochim. Biophys. Acta 1847, 1017-1024. doi: 10.1016/j.bbabio.2015.02.003

Pontier, D., Albrieux, C., Joyard, J., Lagrange, T., and Block, M. A. (2007). Knock-out of the magnesium protoporphyrin IX methyltransferase gene in Arabidopsis. Effects on chloroplast development and on chloroplast-to-nucleus signaling. J. Biol. Chem. 282, 2297-2304. doi: 10.1074/jbc.M610286200

Pöpperl, G., Oster, U., and Rüdiger, W. (1998). Light-dependent increase in chlorophyll precursors during th day-night cycle in tobacco and barley seedlings. J. Plant Physiol. 153, 40-45. doi: 10.1016/S0176-1617(98)80042-4

Rissler, H. M., Collakova, E., DellaPenna, D., Whelan, J., and Pogson, B. J. (2002). Chlorophyll biosynthesis. Expression of a second chl I gene of magnesium chelatase in Arabidopsis supports only limited chlorophyll synthesis. Plant Physiol. 128, 770-779. doi: 10.1104/pp.010625

Ruckle, M. E., Burgoon, L. D., Lawrence, L. A., Sinkler, C., and Larkin, R. M. (2012). Plastids are major regulators of light signaling in Arabidopsis thaliana. Plant Physiol. 159, 366-390. doi: 10.1104/pp.112.193599

Ruckle, M. E., DeMarco, S. M., and Larkin, R. M. (2007). Plastid signals remodel light signaling networks and are essential for efficient chloroplast biogenesis in Arabidopsis. Plant Cell 19, 3944-3960. doi: 10.1105/tpc.107.054312

Ruckle, M. E., and Larkin, R. M. (2009). Plastid signals that affect photomorphogenesis in Arabidopsis thaliana are dependent on GENOMES UNCOUPLED 1 and cryptochrome 1. New Phytol. 182, 367-379. doi: 10.1111/j.1469-8137.2008.02729.x

Ryu, J. S., Kim, J. I., Kunkel, T., Kim, B. C., Cho, D. S., Hong, S. H., et al. (2005). Phytochrome-specific type 5 phosphatase controls light signal flux by enhancing phytochrome stability and affinity for a signal transducer. Cell 120, 395-406. doi: 10.1016/j.cell.2004.12.019

Saini, G., Meskauskiene, R., Pijacka, W., Roszak, P., Sjögren, L. L., Clarke, A. K., et al. (2011). 'happy on norflurazon' (hon) mutations implicate perturbance of plastid homeostasis with activating stress acclimatization and changing nuclear gene expression in norflurazon-treated seedlings. Plant J. 65, 690-702. doi: 10.1111/j.1365-313X.2010.04454.x

Sangster, T. A., Bahrami, A., Wilczek, A., Watanabe, E., Schellenberg, K., McLellan, C., et al. (2007). Phenotypic diversity and altered environmental plasticity in Arabidopsis thaliana with reduced Hsp90 levels. PLoS ONE 2:e648. doi: 10.1371/journal.pone.0000648

Schlicke, H., Hartwig, A. S., Firtzlaff, V., Richter, A. S., Glässer, C., Maier, K., et al. (2014). Induced deactivation of genes encoding chlorophyll biosynthesis enzymes disentangles tetrapyrrole-mediated retrograde signaling. Mol. Plant 7 , 1211-1227. doi: $10.1093 / \mathrm{mp} / \mathrm{ssu} 034$
Schön, A., Krupp, G., Gough, S., Berry-Lowe, S., Kannangara, C. G., and Söll, D. (1986). The RNA required in the first step of chlorophyll biosynthesis is a chloroplast glutamate tRNA. Nature 322, 281-284. doi: 10.1038/322281a0

Staneloni, R. J., Rodriguez-Batiller, M. J., and Casal, J. J. (2008). Abscisic acid, highlight, and oxidative stress down-regulate a photosynthetic gene via a promoter motif not involved in phytochrome-mediated transcriptional regulation. Mol. Plant 1, 75-83. doi: 10.1093/mp/ssm007

Strand, ̊̊, Asami, T., Alonso, J., Ecker, J. R., and Chory, J. (2003). Chloroplast to nucleus communication triggered by accumulation of Mg-protoporphyrinIX. Nature 421, 79-83. doi: 10.1038/nature01204

Sullivan, J. A., and Gray, J. C. (2002). Multiple plastid signals regulate the expression of the pea plastocyanin gene in pea and transgenic tobacco plants. Plant J. 32, 763-774. doi: 10.1046/j.1365-313X.2002.01464.x

Sun, X., Feng, P., Xu, X., Guo, H., Ma, J., Chi, W., et al. (2011). A chloroplast envelope-bound PHD transcription factor mediates chloroplast signals to the nucleus. Nat. Commun. 2:477. doi: 10.1038/ncomms1486

Susek, R., and Chory, J. (1992). A tale of two genomes: role of a chloroplast signal in coordinating nuclear and plastid gene expression. Aust. J. Plant Physiol. 19, 387-399. doi: 10.1071/PP9920387

Susek, R. E., Ausubel, F. M., and Chory, J. (1993). Signal transduction mutants of Arabidopsis uncouple nuclear CAB and RBCS gene expression from chloroplast development. Cell 74, 787-799. doi: 10.1016/0092-8674(93)90459-4

Tadini, L., Pesaresi, P., Kleine, T., Rossi, F., Guljamow, A., Sommer, F., et al. (2016). GUN1 controls accumulation of the plastid ribosomal protein S1 at the protein level and interacts with proteins involved in plastid protein homeostasis. Plant Physiol. 170, 1817-1830. doi: 10.1104/pp.15.02033

Tameshige, T., Fujita, H., Watanabe, K., Toyokura, K., Kondo, M., Tatematsu, K. et al. (2013). Pattern dynamics in adaxial-abaxial specific gene expression are modulated by a plastid retrograde signal during Arabidopsis thaliana leaf development. PLoS Genet. 9:e1003655. doi: 10.1371/journal.pgen.1003655

Tanaka, H., Maruta, T., Tamoi, M., Yabuta, Y., Yoshimura, K., Ishikawa, T., et al. (2015). Transcriptional control of vitamin C defective 2 and tocopherol cyclase genes by light and plastid-derived signals: the partial involvement of GENOMES UNCOUPLED 1. Plant Sci. 231, 20-29. doi: 10.1016/j.plantsci.2014.11.007

Tanaka, K., and Hanaoka, M. (2013). The early days of plastid retrograde signaling with respect to replication and transcription. Front. Plant Sci. 3:301. doi: 10.3389/fpls.2012.00301

Tanaka, R., Kobayashi, K., and Masuda, T. (2011). Tetrapyrrole metabolism in Arabidopsis thaliana. Arabidopsis Book 9:e0145. doi: 10.1199/tab.0145

Tanaka, R., and Tanaka, A. (2007). Tetrapyrrole biosynthesis in higher plants. Annu. Rev. Plant Biol. 58, 321-346. doi: 10.1146/annurev.arplant.57.032905. 105448

Tarahi Tabrizi, S., Sawicki, A., Zhou, S., Luo, M., and Willows, R. D. (2016). GUN4-protoporphyrin IX is a singlet oxygen generator with consequences for plastid retrograde signaling. J. Biol. Chem. 291, 8978-8984. doi: 10.1074/jbc.C116.719989

Terry, M. J., and Smith, A. G. (2013). A model for tetrapyrrole synthesis as the primary mechanism for plastid-to-nucleus signaling during chloroplast biogenesis. Front. Plant Sci. 4:14. doi: 10.3389/fpls.2013.00014

Thomas, J., and Weinstein, J. D. (1990). Measurement of heme efflux and heme content in isolated developing cotyledons. Plant Physiol. 94, 1414-1423. doi: 10.1104/pp.94.3.1414

Verdecia, M. A., Larkin, R. M., Ferrer, J. L., Riek, R., Chory, J., and Noel, J. (2005). Structure of the Mg-chelatase cofactor GUN4 reveals a novel hand-shaped fold for porphyrin binding. PLoS Biol. 3:e151. doi: 10.1371/journal.pbio.0030151

Vinti, G., Hills, A., Campbell, S., Bowyer, J. R., Mochizuki, N., Chory, J., et al. (2000). Interactions between hyl and gun mutants of Arabidopsis, and their implications for plastid/nuclear signalling. Plant J. 24, 883-894. doi: 10.1046/j.1365-313x.2000.00936.x

Voigt, C., Oster, U., Börnke, F., Jahns, P., Dietz, K. J., Leister, D., et al. (2010). Indepth analysis of the distinctive effects of norflurazon implies that tetrapyrrole biosynthesis, organellar gene expression and ABA cooperate in the GUNtype of plastid signalling. Physiol. Plant. 138, 503-519. doi: 10.1111/j.13993054.2009.01343.x

von Gromoff, E. D., Alawady, A., Meinecke, L., Grimm, B., and Beck, C. F. (2008). Heme, a plastid-derived regulator of nuclear gene expression in Chlamydomonas. Plant Cell 20, 552-567. doi: 10.1105/tpc.107.054650 
Voß, B., Meinecke, L., Kurz, T., Al-Babili, S., Beck, C. F., and Hess, W. R. (2011). Hemin and magnesium-protoporphyrin IX induce global changes in gene expression in Chlamydomonas reinhardtii. Plant Physiol. 155, 892-905. doi: 10.1104/pp.110.158683

Waters, M. T., Wang, P., Korkaric, M., Capper, R. G., Saunders, N. J., and Langdale, J. A. (2009). GLK transcription factors coordinate expression of the photosynthetic apparatus in Arabidopsis. Plant Cell 21, 1109-1128. doi: 10.1105/tpc.108.065250

Woodson, J. D., Joens, M. S., Sinson, A. B., Gilkerson, J., Salomé, P. A., Weigel, D., et al. (2015). Ubiquitin facilitates a quality-control pathway that removes damaged chloroplasts. Science 350, 450-454. doi: 10.1126/science.aac7444

Woodson, J. D., Perez-Ruiz, J. M., and Chory, J. (2011). Heme synthesis by plastid ferrochelatase I regulates nuclear gene expression in plants. Curr. Biol. 21, 897-903. doi: 10.1016/j.cub.2011.04.004

Woodson, J. D., Perez-Ruiz, J. M., Schmitz, R. J., Ecker, J. R., and Chory, J. (2013). Sigma factor mediated plastid retrograde signals control nuclear gene expression. Plant J. 73, 1-13. doi: 10.1111/tpj.12011

Xu, X., Chi, W., Sun, X., Feng, P., Guo, H., Li, J., et al. (2016). Convergence of light and chloroplast signals for de-etiolation through ABI4-HY5 and COP1. Nat. Plants 2:16066. doi: 10.1038/nplants.2016.66

Zhang, Z. W., Feng, L. Y., Cheng, J., Tang, H., Xu, F., Zhu, F., et al. (2013). The roles of two transcription factors, $\mathrm{ABI} 4$ and CBFA, in ABA and plastid signalling and stress responses. Plant Mol. Biol. 83, 445-458. doi: 10.1007/s11103-013-0102-8
Zhang, Z. W., Yuan, S., Feng, H., Xu, F., Cheng, J., Shang, J., et al. (2011a). Transient accumulation of $\mathrm{Mg}$-protoporphyrin IX regulates expression of PhANGs - New evidence for the signaling role of tetrapyrroles in mature Arabidopsis plants. J. Plant Physiol. 168, 714-721. doi: 10.1016/j.jplph.2010. 10.016

Zhang, Z. W., Yuan, S., Xu, F., Yang, H., Chen, Y. E., Yuan, M., et al. (2011b). Mg-protoporphyrin, haem and sugar signals double cellular total RNA against herbicide and high-light-derived oxidative stress. Plant Cell Environ. 34, 10311042. doi: 10.1111/j.1365-3040.2011.02302.x

Zhang, Z. W., Zhang, G. C., Zhu, F., Zhang, D. W., and Yuan, S. (2015). The roles of tetrapyrroles in plastid retrograde signaling and tolerance to environmental stresses. Planta 242, 1263-1276. doi: 10.1007/s00425-015-2384-3

Conflict of Interest Statement: The author declares that the research was conducted in the absence of any commercial or financial relationships that could be construed as a potential conflict of interest.

Copyright (C) 2016 Larkin. This is an open-access article distributed under the terms of the Creative Commons Attribution License (CC BY). The use, distribution or reproduction in other forums is permitted, provided the original author(s) or licensor are credited and that the original publication in this journal is cited, in accordance with accepted academic practice. No use, distribution or reproduction is permitted which does not comply with these terms. 